Bundesgesundheitsbl 2019 · 62:352-376 https://doi.org/10.1007/s00103-019-02882-5 (c) Springer-Verlag GmbH Deutschland, ein Teil von Springer Nature 2019

\section{Electronic supplementary material}

The online version of this article (https:// doi.org/10.1007/s00103-019-02882-5) contains supplementary material, which is available to authorized users.

\section{Summary}

The STIKO recommends vaccination with the adjuvanted herpes zoster subunit (HZ/ su) inactivated vaccine for the preven-

\footnotetext{
A. Siedler and J. Koch have contributed equally to the publication.

E. Garbe, R. von Kries, T. Ledig, T. Mertens, F. Zepp, K. Überla, are members of the German Standing Committee on Vaccination (STIKO). This report is a translation of the original publication in German language in Epidemiologisches Bulletin 50/2018, available at: https:// www.rki.de/DE/Content/Infekt/EpidBull/ Archiv/2018/Ausgaben/50_18.pdf?_blob=publicationFile.
}

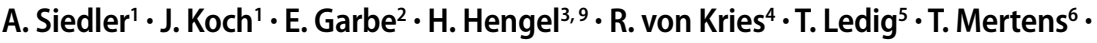 \\ F. Zepp $p^{7} \cdot$ K. Überla ${ }^{8}$ \\ 'Immunization Unit, Robert Koch Institute, Berlin, Germany \\ ${ }^{2}$ Bremen Institute for Prevention Research and Social Medicine, University of Bremen, Bremen, Germany \\ ${ }^{3}$ Institute of Virology, Medical Center-University of Freiburg, Faculty of Medicine, Freiburg, Germany \\ ${ }^{4}$ Institute of Social Paediatrics and Adolescent Medicine, Ludwig-Maximilians University of Munich, Munich, \\ Germany \\ ${ }^{5}$ General Practice, Ditzingen, Germany \\ ${ }^{6}$ Institute of Virology, Ulm University Medical Center, Ulm, Germany \\ ${ }^{7}$ Department of Pediatrics and Adolescent Medicine, University Medicine Mainz, Mainz, Germany \\ ${ }^{8}$ Institute of Clinical and Molecular Virology, University of Erlangen-Nuernberg, Erlangen, Germany \\ ${ }^{9}$ German Consulting Laboratory for HSV and VZV, Medical Center - University of Freiburg, Freiburg, Germany
}

\title{
Background paper to the decision to recommend the vaccination with the inactivated herpes zoster subunit vaccine
}

\author{
Statement of the German Standing \\ Committee on Vaccination (STIKO) at the \\ Robert Koch Institute
}

tion of herpes zoster (HZ) and postherpetic neuralgia $(\mathrm{PHN})$ for all people age 60 years and over (standard vaccination).

This recommendation takes into account the good efficacy of the vaccine, the anticipated period of protection it provides, and the increased risk of severe $\mathrm{HZ}$ disease and post-zoster pain in individuals age 60 years and over. Models of the epidemiological effects of vaccination show that administering the $\mathrm{HZ} /$ su vaccine at age 60 years has the greatest effect in preventing all $\mathrm{HZ}$ cases, and administering the vaccine at age 70 years showed the greatest effect in preventing $\mathrm{PHN}$, in a vaccinated cohort. According to the results of a health economics model, the lowest cost per quality-adjusted life year (QALY) would be achieved with vaccination at age 65 years. The number of people who need to be vaccinated (number needed to vaccinate, $\mathrm{NNV}$ ) to prevent one case of $\mathrm{HZ}$ is the same for both vaccination ages (60 and 65 years). In light of the fact that preventing $\mathrm{HZ}$ is the key prerequisite to prevent- ing complications and late sequelae such as PHN, 60 years of age is considered the most favorable age for vaccination, to prevent both $\mathrm{HZ}$ and its complications.

The STIKO also recommends vaccination against $\mathrm{HZ}$ and $\mathrm{PHN}$ with the HZ/ su inactivated vaccine for all people from the age of 50 years who have an elevated risk of $\mathrm{HZ}$ and $\mathrm{PHN}$ owing to increased health risks as a consequence of an underlying disease or immunosuppression (indication-based vaccination). This group includes e.g. people with congenital or acquired immunodeficiency or immunosuppression, HIV infection, rheumatoid arthritis, systemic lupus erythematosus, chronic inflammatory bowel disease, chronic obstructive pulmonary disease (COPD) or bronchial asthma, chronic renal disease, diabetes mellitus.

The efficacy and safety of the vaccine for patients with impaired immune systems have been demonstrated in numerous studies. Stratified data analyses on the efficacy of the vaccine have shown no difference in 
age group (years)
Fig. $1<$ Incidence of herpes zoster $(H Z)$ by age and sex and proportion of postherpetic neuralgia $(P H N)$ among $\mathrm{HZ}$ cases in Germany [5, 43] comparison to overall efficacy for patients with an underlying disease, e.g., rheumatoid arthritis, chronic renal disease, COPD, or diabetes mellitus, who were enrolled in vaccine marketing authorization studies.

\section{Introduction}

Herpes zoster (HZ) and its most frequent complication, postherpetic neuralgia $(\mathrm{PHN})$, place a large disease burden and limitations on the life quality of people affected in Germany. In March 2018, the European regulatory authority approved an adjuvanted $\mathrm{HZ}$ subunit $(\mathrm{HZ} / \mathrm{su})$ vaccine for the prevention of $\mathrm{HZ}$ and PHN from the age of 50 years (Shingrix $^{\oplus}$; Glaxo SmithKline (GSK), Rixensart, Belgium); this vaccine contains the adjuvant $\mathrm{ASO}_{\mathrm{B}}$ and recombinant varicella zoster virus (VZV) glycoprotein $\mathrm{E}(\mathrm{gE})$. This background paper summarizes the data basis used by the STIKO in its decision regarding standard vaccination and indication-based vaccination with the adjuvanted $\mathrm{HZ} / \mathrm{su}$ inactivated vaccine. The present information is also based on the data already used in the STIKO decision on the live HZ vaccine in its 2017/2018 recommendations [1]. For example, the current disease burden of HZ in Germany had already been determined according to the STIKO standard operating procedure (SOP) [2]. This was followed by systematic reviews on the efficacy and safety of the $\mathrm{HZ} /$ su inactivated vaccine and evaluations of the data on the vaccine induced period of protection. A mathematical model of the potential epidemiological effects of $\mathrm{HZ}$ vaccination in Germany was conducted, which served as the basis for a health economics evaluation of potential vaccination strategies. This background paper includes a comprehensive appendix as electronic supplementary material containing further information on the systematic reviews conducted as well as on the decision process by the STIKO, which is available for download at https:// link.springer.com/journal/volumesAndIssues/103.

\section{Causes and symptoms}

Detailed information on the etiology, symptoms, localization, complications, and risk factors of $\mathrm{HZ}$ was recently published in a STIKO background paper presenting the rationale of the STIKO decision against recommendation of the live $H Z$ vaccine as a standard vaccination [1, 3]. For that reason a detailed description is not presented here.

\section{Epidemiology}

Data on the epidemiology of $\mathrm{HZ}$ were also published in the aforementioned background paper [1, 3]. These will be summarized again here, and complemented with data on the epidemiology of PHN and other complications of $\mathrm{HZ}$, as well as a description of the risk factors of $\mathrm{HZ}$ and PHN.

In Germany, it is estimated that more than 300,000 people develop HZ each year, and that number is increasing. The risk of developing $\mathrm{HZ}$ is age dependent. From age 10 to 44 years, the incidence of $\mathrm{HZ}$ is 4/1000 person-years (PY) [4]; from age 50 years, the incidence rises steadily from around 6/1000 PY to more than
$13 / 1000$ PY from age 70 years $[4,5]$. The incidence of $\mathrm{HZ}$ is higher among women than among men in every age group (• Fig. 1).

Hospitalizations owing to $\mathrm{HZ}$ and its complications also increase with age. The reported incidence of $\mathrm{HZ}$ cases treated in the hospital is $0.13 / 1000 \mathrm{PY}$ in the age group 50-54 years, and rises to around $1 / 1000$ PY from the age of 80 years [5]. According to hospital diagnosis statistics, the annual number of $\mathrm{HZ}$ cases among people age $>50$ years treated in hospitals has approximately doubled in the past 10 years, with nearly 20,000 cases in 2015. Complications are recorded for more than $60 \%$ of patients hospitalized with $\mathrm{HZ}$, the most frequent being $\mathrm{HZ}$ with clinical symptoms affecting the nervous system and herpes zoster ophthalmicus. The percentage of hospitalized patients with complications remains constant with increasing age in people age 50 years and older, but the number of patients treated in the hospital who develop complications rises with age (http://www.gbe-bund.de/gbe10/abrechnung.prc_abr_test_logon?p_uid=gast\&p_ aid $=0 \& p \_$knoten $=$VR\&p_sprache $=$D\&p_ suchstring $=\mathrm{g} \% \mathrm{FCrtel}$ rose). An analysis of data from insured individuals also showed an increase with age for all other $\mathrm{HZ}$ complications and multiple complications, except for HZ meningitis [4].

The risk of $\mathrm{PHN}$ following $\mathrm{HZ}$ rises steadily with age. In the aforementioned analysis of data from insured individuals, the percentage of PHN cases among all $\mathrm{HZ}$ cases in the age group 50-54 years was approximately $12 \%$, and this increased to $>20 \%$ with age until $80-84$ years [4]. In a more conservative estimate based on out- 
patient diagnosis invoicing, the percentage of PHN among people age 50-59 years was $3 \%$, and around $8 \%$ in those age 70 years or more [5].

Immunosuppression and other underlying diseases have been described as additional risk factors for $\mathrm{HZ}$ and its complications. In an analysis of clinical data from Germany, the percentage of patients who developed PHN in all age groups was 36\% higher among those with immunosuppression than among patients with a healthy immune system; in those age 50 years and over, this rate was $18 \%$ higher [4]. Patients with immunosuppression were defined in this study as those with HIV infection, malignant tumors, organ or stem-cell transplant recipients, and patients with other reasons for immunosuppression.

In a systematic review covering 84 mostly retrospective cohort studies conducted mainly in North America, Europe, and Asia from January 2003 to February 2017, the following comorbidities were identified as risk factors for $\mathrm{HZ}(\mathrm{RR}$, relative risk) [6]:

- Rheumatoid arthritis: RR 1.19-2.40

- Systemic lupus erythematosus:

RR 1.29-4.11

- Inflammatory bowel disease:

RR 1.26-1.50

- Chronic renal disease: RR 1.14-1.60

- COPD: RR 1.17-1.68

- Asthma: RR 1.11-1.70

- Diabetes mellitus: RR 1.02-1.68

In another systematic review, the risk of $\mathrm{HZ}$ was examined in patients with rheumatoid arthritis, psoriasis, SLE, or chronic inflammatory bowel diseases, as well as those who had received immunosuppressive therapy with biologics or with so-called non-biologic disease modifying anti-rheumatic drugs (nbDMARDS) [7]. During the time frame 1946-2016, a total of 40 randomized controlled trials (RCTs) including 20,136 patients and 19 observational studies with a total of 810,939 patients were identified. In the meta-analyses of both RCTs (odds ratio $(\mathrm{OR})=1.71$, 95\% confidence interval (CI) 1.11-2.64) and observational studies $(\mathrm{OR}=1.58,95 \%$ CI 1.39-1.81), an increased risk of HZ was found among patients who received biologics therapy, especially among those who had been treated with non-TNF- $\alpha$ antagonists $(\mathrm{OR}=2.19$, 95\% CI 1.20 4.02). In contrast, patients who were treated with TNF- $\alpha$ antagonists did not have a significantly higher HZ risk. Patients who received high-dosage nbDMARDS or high-dose corticosteroids also showed an increased risk.

One study from the United Kingdom (UK) analyzed the risk of PHN using data from a routine database of patients with $\mathrm{HZ}$ in primary care [6]. The study findings showed that the incidence of PHN was markedly higher in the following risk groups: patients with leukemia (14.4\%), lymphoma $(12.1 \%)$, myeloma $(17 \%)$, rheumatoid arthritis (9.1\%), SLE (9.4\%), COPD (13.2\%), and chronic renal disease $(10.6 \%)$, and patients who received highdose corticosteroid therapy $(14.5 \%)$ and homologous stem-cell therapy (29.4\%).

Patients with immunosuppression and other severe underlying diseases (particularly autoimmune diseases) are at higher risk of developing $\mathrm{HZ}$ than those with healthy immune systems at any age. These individuals also more frequently experience severe progression or complications of HZ. For this reason, evidence on the efficacy and safety of vaccination with the $\mathrm{HZ} /$ su inactivated vaccine was systematically reviewed for these populations (see Sect. 11.6).

\section{Herpes zoster subunit inactivated vaccine}

An adjuvanted $\mathrm{HZ} / \mathrm{su}$ inactivated vaccine (Shingrix ${ }^{\oplus}$, manufactured by GSK, Belgium) was approved for use in Europe on 21 March 2018 by the European Medicines Agency [7]; the vaccine became available in Germany in May 2018. This immunogenic vaccine contains recombinant surface glycoprotein E $(50 \mu \mathrm{g})$ of VZV. The HZ/su inactivated vaccine also contains the adjuvants $\mathrm{AS} 01_{\mathrm{B}}$, consisting of monophosphoryl lipid A (MPL) from Salmonella minnesota and Quillaja saponaria Molina fraction 21 (QS-21), a surface-active substance from the South American soap bark tree.

The adjuvant contains elements that enhance the $\mathrm{CD} 4^{+} \mathrm{T}$ cell and humoral immune response [8]. Thus, the vaccine can trigger a strong, cell-mediated immune response in individuals whose adaptive im- mune system is impaired, e.g., owing to immunosenescence or for other reasons of immunosuppression. The same adjuvant was used for the first time in a malaria candidate vaccine for children. There is no experience in using this adjuvant outside of clinical trials.

One dose $(0.5 \mathrm{~mL})$ of the reconstituted $\mathrm{HZ} /$ su inactivated vaccine (powder and solvent for producing a suspension for injection) contains $50 \mu \mathrm{g}$ VZV gE antigen, $50 \mu \mathrm{g}$ MPL, and $50 \mu \mathrm{g}$ QS-21. Additional ingredients in the vaccine are as follows. The powder (gE antigen) contains saccharose, polysorbate 80 , sodium dihydrogen phosphate dihydrate, and dipotassium phosphate; the suspension $\left(\mathrm{AS} 01_{\mathrm{B}}\right.$ adjuvant system) contains dioleoylphosphatidylcholine, cholesterol, sodium chloride, disodium phosphate (anhydrous), potassium dihydrogenphosphate, and water for injection purposes. The vaccine does not contain any thimerosal or other preservatives. The $\mathrm{HZ} / \mathrm{su}$ inactivated vaccine is approved for the prevention of $\mathrm{HZ}$ and HZ-attributable PHN in adults 50 years of age and older. The vaccination series consists of two vaccinations administered i. $\mathrm{m}$. at least 2 months apart. The time frame for administration of the second dose can be extended up to 6 months after the first vaccine dose. The need for and optimal time frame of vaccination boosters after basic immunization is completed is not yet known. The safety and efficacy of the vaccine in children and adolescents is not yet known. Data are available on simultaneous administration with other vaccines, addressed in Sect. 10.2. Vaccination is contraindicated in cases of hypersensitivity to any of the ingredients in the vaccine. No data are available on the administration of the $\mathrm{HZ} /$ su inactivated vaccine to pregnant women. In several studies with patients receiving immunosuppressive therapy or patients with immunodeficiency disease, the vaccine has been demonstrated to be immunogenic and well tolerated [9].

\section{Vaccination aims}

The primary aims of vaccination with the adjuvanted $\mathrm{HZ} / \mathrm{su}$ subunit vaccine are a reduction in the frequency of $\mathrm{HZ}$ and prevention of complications and $\mathrm{HZ}$ sequelae, such as PHN, in adults age 60 


\section{Table 1 Hierarchy of patient-relevant endpoints for the evaluation of efficacy and safety of the herpes zoster subunit inactivated vaccine}

\begin{tabular}{|c|c|c|c|c|c|}
\hline $\begin{array}{l}\text { Type of } \\
\text { endpoint }\end{array}$ & Population & Intervention & Comparator & Endpoints (outcomes) & $\begin{array}{l}\text { Assessment of } \\
\text { the significance } \\
\text { of endpoints } \\
\text { for a decision }^{\mathrm{a}}\end{array}$ \\
\hline \multirow[t]{4}{*}{ Efficacy } & \multirow[t]{4}{*}{ Adults $\geq 50$ years $^{\mathrm{b}}$} & \multirow{4}{*}{$\begin{array}{l}\text { Vaccination with } \\
\mathrm{HZ} / \text { su inactivated } \\
\text { vaccine }\end{array}$} & \multirow{4}{*}{$\begin{array}{l}\text { No vaccination; Placebo vaccination; } \\
\text { Other vaccination }\end{array}$} & Herpes zoster (HZ) & 9 \\
\hline & & & & $\begin{array}{l}\text { Postherpetic neuralgia } \\
(\mathrm{PHN})\end{array}$ & 8 \\
\hline & & & & $\begin{array}{l}\text { Other complications } \\
\text { (including death) }\end{array}$ & 7 \\
\hline & & & & Hospitalization & 7 \\
\hline \multirow[t]{4}{*}{ Safety } & \multirow[t]{4}{*}{ Adults $\geq 50$ years $^{b}$} & \multirow{4}{*}{$\begin{array}{l}\text { Vaccination with } \\
\mathrm{HZ} / \text { su inactivated } \\
\text { vaccine }\end{array}$} & \multirow{4}{*}{$\begin{array}{l}\text { No vaccination; Placebo vaccination; } \\
\text { Other vaccination }\end{array}$} & Local reactions, not severe & 3 \\
\hline & & & & Severe local reactions & 7 \\
\hline & & & & $\begin{array}{l}\text { Systemic reactions, not } \\
\text { severe }\end{array}$ & 5 \\
\hline & & & & Severe systemic reactions & 8 \\
\hline \multicolumn{6}{|c|}{$\begin{array}{l}\text { aScale from 1-9: essential/critical (7-9 points), important ( } 4-6 \text { points), or of limited significance (1-3 points). Each endpoint must be assessed on its own. The same score } \\
\text { can be assigned to multiple endpoints, as different endpoints can be equally significant } \\
\text { bAge group should be selected according to the modeling results }\end{array}$} \\
\hline
\end{tabular}

years and over. The longest possible protection for vaccinated individuals should be achieved.

The aim of indication-based vaccination is a reduction in the frequency of $\mathrm{HZ}$ and prevention of complications and $\mathrm{HZ}$ sequelae in populations with an elevated risk of $\mathrm{HZ}$, according to the approved age at vaccination of 50 years or over.

\section{Method of searching and assessing the quality of evidence}

The evidence on efficacy and safety of the $\mathrm{HZ} / \mathrm{su}$ inactivated vaccine was reviewed and assessed for quality according to the STIKO SOP for the systematic development of vaccination recommendations [2]. After the STIKO formulated the primary aims of $\mathrm{HZ}$ vaccination, and following the methods of the Grading of Recommendations Assessment, Development and Evaluation (GRADE) working group, patient-relevant endpoints of $\mathrm{HZ}$ vaccination were defined. The endpoints HZ, PHN, other complications (including death), and hospitalization were selected for vaccine efficacy. The endpoints for vaccine safety were non-severe local reactions, severe local reactions, non-severe systemic reactions, and severe systemic reactions. All endpoints were assessed on a scale of 1-9 as essential/critical (7-9 points), impor- tant (4-6 points), or of limited significance (1-3 points) in the decision on a vaccination recommendation by working group members (• Table 1$)$.

To identify clinical studies on vaccine safety and efficacy, systematic literature research following the requirements set forth in the PRISMA statement (Preferred Reporting Items for Systematic Reviews and Meta-Analyses) was conducted using the following databases and taking patient-relevant endpoints into account: MEDLINE; EMBASE, BIOSIS Previews, SciSearch, Cochrane Central Register of Controlled Trials, Cochrane Database of Systematic Reviews, GLOBAL Health [10]. The complete search strategies, flowcharts, and inclusion and exclusion criteria are provided in the appendix (last search date: 4 November 2017). Additionally, reference lists of the studies included and the reviews identified were screened for other potentially relevant studies. No limitations were placed on publication status or language.

The literature research and data extraction were conducted by two independent investigators (AS, JK). The relevant study characteristics of the original studies that fulfilled the inclusion criteria were recorded using a standardized extraction form and their internal and external validity was evaluated. Discrepancies between the two investigators were discussed until consen- sus was reached. The Cochrane risk of bias tool was used to assess the risk of bias in randomized controlled trials (RCTs) [11].

We entered the extracted data on patient-relevant endpoints from the included studies into RevMan (version 5.2) review management software, and RRs and corresponding 95\% CIs of the vaccine group compared with the placebo group were calculated for the respective endpoints. If more than one study was available, a meta-analysis was conducted and the pooled estimates determined. If heterogeneity was present (assessed using the $\mathrm{I}^{2}$ statistic), a random-effects model was used; otherwise, the data were summarized using a fixed-effects model. Using the pooled RR, the formula $((1-R R) \cdot 100)$ was applied to calculate the vaccine efficacy or effectiveness or the risk of adverse side effects of vaccination.

To compile the GRADE evidence profile, pooled data from the endpoints defined as "critical" and "important" were entered into the GRADE profiler (version 3.6), and the quality of evidence in all included studies were assessed for each endpoint, according to the following aspects: study design, heterogeneity and precision, indirect evidence, effect size, and publication bias. Assessment of the overall quality of evidence across all endpoints was conducted using the lowest quality of evidence in those endpoints defined as "critical" $[12,13]$. 


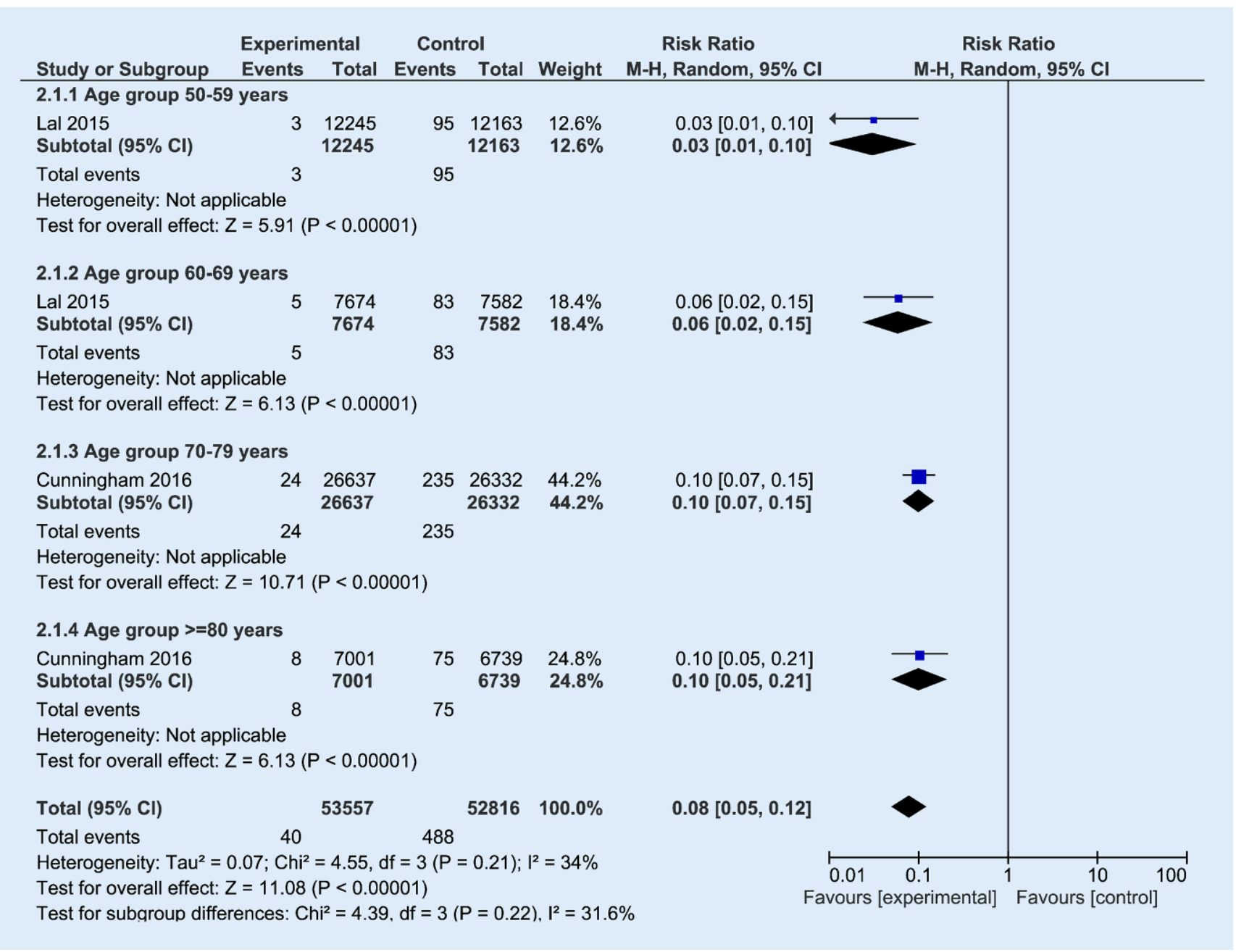

Fig. $2 \Delta$ Efficacy of $\mathrm{HZ} /$ su inactivated vaccine in preventing $\mathrm{HZ}$ in various age groups ( $\geq 50-59$ years, $\geq 60-69$ years, $\geq 70-79$

years, $\geq 80$ years); information from the cumulative follow-up periods (total) in person-years [14, 15]

\section{Vaccine efficacy and period of protection}

Vaccine efficacy (VE) is defined as the ability of a vaccine to prevent the incidence of a disease (e.g., HZ) or disease-related endpoints (such as PHN) in clinical studies under optimal and controlled conditions. These conditions are normally met in RCTs. Two RCTs were included in the systematic review on efficacy of the $\mathrm{HZ} / \mathrm{su}$ inactivated vaccine; these are presented in greater detail below $[14,15]$. The included RCTs had a low risk of bias.

\subsection{Zoster efficacy study in persons $\geq 50$ years $(Z O E-50)$}

The ZOE-50 study (ClinicalTrials.gov, NCT01165177) was a double blind, placebo-controlled multicenter study to verify efficacy of the $\mathrm{HZ} / \mathrm{su}$ inactivated vaccine in protecting adults age 50 years and above against HZ [15]. The study was conducted in 18 countries in Europe, North America, Latin America, and Asia/Australia. Participants age 50 years and older were recruited at a ratio of 1:1 for the vaccine and placebo arms. The following exclusion criteria were applied: a medical history of HZ, previous vaccination against VZV or HZ, immunosuppression owing to a disease (e. g., malignoma or HIV infection) or immunosuppressive therapy, allergy to one of the components of the vaccine, severe existing underlying disease with a survival time of $<4$ years, simultaneous participation in another clinical trial, administration of another study drug (medicinal product or vaccine) within 30 days before study initiation, administration of immunoglobulins or blood products within 90 days before study initiation, other planned vaccinations within 30 days before study initiation, and acute illness or fever at the time of recruitment. Female participants were excluded if they were pregnant or nursing or planning to become pregnant.

Each study participant received two doses of $0.5 \mathrm{~mL} \mathrm{HZ/su} \mathrm{inactivated} \mathrm{vac-}$ cine or placebo ( $0.9 \%$ saline solution) injected i.m. with an interval of 2 months. Because the solutions differed in appearance, the injection solution was prepared and administered by research assistants who were not involved in the assessment of the study results in any way. Study participants were monitored for a period of at least 30 months after receiving the second vaccine dose via monthly contacts and annual visits. The primary aim of the study was to investigate $\mathrm{VE}$ in protecting against 
Table 2 Efficacy of the $\mathrm{HZ} /$ su inactivated vaccine against $\mathrm{HZ}$ and PHN in the ZOE-50 and ZOE-70 studies and in the pooled cohort, according to age groups (ITT and modified analysis) [14, 15]

\begin{tabular}{|c|c|c|c|c|c|c|c|}
\hline \multicolumn{8}{|c|}{ Prevention of HZ (n) } \\
\hline $\begin{array}{l}\text { Age group, } \\
\text { years }\end{array}$ & $\begin{array}{l}\text { Vaccine } \\
\text { group }\end{array}$ & HZ cases & $\begin{array}{l}\mathrm{HZ} \text { incidence } \\
\text { cases/1000 PY }\end{array}$ & Placebo arm & $\mathrm{HZ}$ cases & $\begin{array}{l}\mathrm{HZ} \text { incidence } \\
\text { cases } / 1000 \mathrm{PY}\end{array}$ & HZ VE (95\% Cl), \% \\
\hline \multicolumn{8}{|l|}{ ZOE-50 (ITT) } \\
\hline $50-59$ & 3645 & 3 & 0.2 & 3644 & 95 & 7.8 & $96.9(90.6-99.4)$ \\
\hline $60-69$ & 2244 & 5 & 0.7 & 2246 & 83 & 10.9 & $94.1(85.6-98.1)$ \\
\hline$\geq 70$ & 1809 & 1 & 0.2 & 1823 & 57 & 10.2 & $98.3(89.9-100)$ \\
\hline Overall & 7698 & 9 & 0.4 & 7713 & 235 & 9.3 & $96.2(92.7-98.3)$ \\
\hline \multicolumn{8}{|c|}{ ZOE-50 (modified analysis) } \\
\hline $50-59$ & 3492 & 3 & 0.3 & 3525 & 87 & 7.8 & $96.6(89.6-99.3)$ \\
\hline $60-69$ & 2141 & 2 & 0.3 & 2166 & 75 & 10.8 & $97.4(90.1-99.7)$ \\
\hline$\geq 70$ & 1711 & 1 & 0.2 & 1724 & 48 & 9.4 & $97.9(87.9-100.0)$ \\
\hline Overall & 7344 & 6 & 0.3 & 7415 & 210 & 9.1 & $97.2(93.7-99.0)$ \\
\hline \multicolumn{8}{|l|}{ ZOE-70 (ITT) } \\
\hline $70-79$ & 5414 & 22 & 1.0 & 5420 & 181 & 8.7 & $88.0(81.3-92.7)$ \\
\hline$\geq 80$ & 1536 & 8 & 1.4 & 1530 & 59 & 10.9 & $86.9(72.4-94.6)$ \\
\hline Overall & 6950 & 30 & 1.1 & 6950 & 240 & 9.1 & $87.7(82.0-92.0)$ \\
\hline \multicolumn{8}{|c|}{ ZOE-70 (modified analysis) } \\
\hline 70-79 & 5114 & 17 & 0.9 & 5189 & 169 & 8.8 & $90.0(83.5-94.4)$ \\
\hline$\geq 80$ & 1427 & 6 & 1.2 & 1433 & 54 & 11.0 & $89.1(74.6-96.2)$ \\
\hline Overall & 6541 & 23 & 0.9 & 6622 & 223 & 9.2 & $89.8(84.2-93.7)$ \\
\hline \multicolumn{8}{|c|}{ Pooled analysis ZOE-50 + ZOE-70 (ITT) } \\
\hline 70-79 & 6837 & 24 & 0.9 & 6856 & 235 & 8.9 & $89.9(84.6-93.7)$ \\
\hline$\geq 80$ & 1921 & 8 & 1.1 & 1917 & 75 & 11.1 & $89.7(78.6-95.8)$ \\
\hline Overall & 8758 & 32 & 1.0 & 8773 & 310 & 9.4 & $89.9(85.4-93.2)$ \\
\hline \multicolumn{8}{|c|}{ Pooled analysis ZOE-50 + ZOE-70 (modified analysis) } \\
\hline $70-79$ & 6468 & 19 & 0.8 & 6554 & 216 & 8.9 & $91.3(86.0-94.4)$ \\
\hline$\geq 80$ & 1782 & 6 & 1.0 & 1792 & 68 & 11.1 & $91.4(80.2-97.0)$ \\
\hline Overall & 8250 & 25 & 0.8 & 8346 & 284 & 9.3 & $91.3(86.8-94.5)$ \\
\hline
\end{tabular}

HZ from the age of 50 years. The secondary aim was to show evidence of efficacy in the defined age groups. VE was identified as a reduction in the risk of developing HZ. Efflorescence suspected as attributable to HZ occurring after the second vaccine dose was examined by the study investigators. In each suspected case, swabs were taken from three lesions to verify the HZ diagnosis via PCR. The lower detection limit was $10 \mathrm{VZV}$ DNA copies. If no examination material was available for laboratory diagnosis, $\mathrm{HZ}$ diagnosis was made by a five-member team of experts based on the clinical picture, photographs of the lesions, and disease progression.
Between August 2010 and July 2011, a total 16,160 study participants were recruited and stratified according to region and age (age groups: 50-59, 60-69, and $\geq 70$ years). A total 749 participants were excluded from the study analysis, mostly owing to good clinical practice guideline violations. The remaining 15,411 study participants were included in the intention-to-treat (ITT) analysis, 7698 in the vaccine arm, and 7713 in the placebo arm. The percentage in each age group was identical in both arms: $47 \%$ of participants were $50-59$ years old, $29 \%$ were age 60-69 years, and $23 \%$ were age 70 years and over. The mean age at study initiation was 62.3 years. Demographic characteristics were congruent between the two groups. Most participants were from Europe (51.2\%), white $(71.8 \%)$, and female $(61.2 \%)$. The mean follow-up period was 3.2 years.

In the ITT analysis, VE in protecting against $\mathrm{HZ}$ from the age of 50 years was $96.2 \%$ (95\% CI 93.0-98.0\%). HZ incidence in the vaccine arm was 0.4 cases/1000 PY, vastly lower than in the placebo arm (09.3/1000 PY). The point estimates of age-specific VE in protecting against $\mathrm{HZ}$ were at a similarly high level, but the confidence intervals were broader. This is especially true for the two highest age groups, as the number of par- 
Table 2 (continued)

\begin{tabular}{|c|c|c|c|c|c|c|c|}
\hline \multicolumn{8}{|c|}{ Prevention of PHN ( $n$ ) } \\
\hline $\begin{array}{l}\text { Age group, } \\
\text { years }\end{array}$ & $\begin{array}{l}\text { Vaccine } \\
\text { group }\end{array}$ & $\begin{array}{l}\text { PHN } \\
\text { cases }\end{array}$ & $\begin{array}{l}\text { PHN incidence } \\
\text { cases } / 1000 \mathrm{PY}\end{array}$ & Placebo arm & PHN cases & $\begin{array}{l}\text { PHN incidence } \\
\text { cases } / 1000 \mathrm{PY}\end{array}$ & PHN VE ( $95 \% \mathrm{Cl}), \%$ \\
\hline \multicolumn{8}{|c|}{ Pooled analysis ZOE-50 + ZOE-70 (ITT) } \\
\hline $50-59$ & 3644 & 0 & 0.0 & 3642 & 9 & 0.6 & $100(49.1-100)$ \\
\hline $60-69$ & 2243 & 0 & 0.0 & 2245 & 3 & 0.3 & $100(-145.2-100)$ \\
\hline $70-79$ & 6837 & 4 & 0.1 & 6856 & 31 & 1.2 & $87.0(63.3-96.7)$ \\
\hline$\geq 80$ & 1921 & 4 & 0.6 & 1917 & 7 & 1.0 & $43.0(-124.3-87.8)$ \\
\hline$\geq 50$ overall & 14645 & 8 & 0.1 & 14660 & 50 & 0.9 & $83.9(65.8-93.5)$ \\
\hline$\geq 70$ overall & 8758 & 8 & 0.2 & 8773 & 38 & 1.1 & $78.9(54.0-91.5)$ \\
\hline \multicolumn{8}{|c|}{ Pooled analysis ZOE-50 + ZOE-70 (modified) } \\
\hline $50-59$ & 3491 & 0 & 0.0 & 3523 & 8 & 0.6 & $100(40.8-100)$ \\
\hline $60-69$ & 2140 & 0 & 0.0 & 2166 & 2 & 0.2 & $100(-442.9-100)$ \\
\hline $70-79$ & 6468 & 2 & 0.1 & 6554 & 29 & 1.2 & $93.0(72.4-99.2)$ \\
\hline$\geq 80$ & 1782 & 2 & 0.3 & 1792 & 7 & 1.1 & $71.6(-51.6-97.1)$ \\
\hline$\geq 50$ overall & 13881 & 4 & 0.1 & 14035 & 46 & 0.9 & $91.2(75.9-97.7)$ \\
\hline$\geq 70$ overall & 8250 & 4 & 0.1 & 8346 & 36 & 1.2 & $88.8(68.7-97.1)$ \\
\hline
\end{tabular}

$H Z$ herpes zoster, $P H N$ postherpetic neuralgia, $P Y$ person-years, VE vaccine efficacy, ITT intention-to-treat, $C I$ confidence interval. ITT all participants successfully recruited and included according to protocol, and received at least one vaccination. Modified analysis exclusion of participants who either did not receive a second vaccine dose or who received the wrong vaccine or a confirmed $\mathrm{HZ}$ diagnosis less than 30 days after the second vaccine dose.

ticipants was markedly lower (• Fig. 2; - Table 2).

In modified analyses, those study participants from the ITT group who did not receive a second vaccine dose or who received the wrong vaccine or an $\mathrm{HZ}$ diagnosis fewer than 30 days after the second vaccine were excluded. In these modified investigation cohorts, VE overall and for each age group was slightly higher than the levels in the ITT group (• Table 2 ).

\subsection{Zoster efficacy study in persons $\geq 70$ years $(\mathrm{ZOE}-70)$}

The ZOE-70 study (ClinicalTrials.gov, NCT01165229) was initiated to examine the efficacy and safety of the HZ/su inactivated vaccine in protecting against $\mathrm{HZ}$ and PHN in adults age 70 years and older, and to conduct a pooled analysis with the results of the ZOE-50 study [14]. The ZOE-70 study was also a double-blind, placebo-controlled study conducted at the same study centers as the ZOE-50 trial and with a study design identical to that of ZOE-50 with regard to inclusion and exclusion criteria, randomization, blinding, stratification according to age and region, and vaccination regimen.
The primary aim of the ZOE-70 study was to investigate VE of the $\mathrm{HZ} /$ su inactivated vaccine in protecting against $\mathrm{HZ}$ in people age $\geq 70$ years. The pooled analysis included study participants age $\geq 70$ years from both studies (ZOE-50 + ZOE70) and had the primary study aim to examine VE against both $\mathrm{HZ}$ and $\mathrm{PHN}$ in this age group. The secondary study aim of the pooled analysis was to determine VE against $\mathrm{PHN}$ in adults age $\geq 50$ years and to evaluate reactogenicity and safety.

The criteria for suspicion and diagnosis of $\mathrm{HZ}$ were identical to those in the $\mathrm{ZOE}$ 50 study. To monitor the occurrence of PHN, all study participants with $\mathrm{HZ}$ were asked to report to the study center regularly for examination. In addition, they were asked to keep a pain diary every day for 28 days and weekly thereafter, in which they documented their pain score, from 0 (no pain) to 10 (severest pain). Entries were to be completed for at least 90 days after the occurrence of $\mathrm{HZ}$ efflorescence and until the patient was pain free for 4 weeks. PHN was defined as pain with a score $\geq 3$ that continued or developed more than 90 days after the occurrence of rash.

A total of 14,816 study participants were recruited between August 2010 and
July 2011. A total of 916 participants were excluded from the study analysis, mostly owing to good clinical practice guideline violations. The remaining 13,900 study participants were included in the (ITT) analysis, 6950 each in the vaccine and placebo arm. The demographic attributes between the vaccine and placebo arms of the ZOE-70 study corresponded roughly with one another. Most participants were from Europe (55\%), white (76.9\%), and female $(54.9 \%)$. The mean age of participants in the ZOE-70 study at study initiation was 75.6 years (range: 62-96 years). In total, 3066 participants were $\geq 80$ years old $(22.1 \%)$ and 76 participants were $\geq 90$ years old $(0.5 \%)$. The mean follow-up period was 3.7 years.

In the ITT analysis of the ZOE-70 study, VE in protecting against $\mathrm{HZ}$ was $87.7 \%$ (95\% CI $82.0 \%-92.0 \%)$. HZ incidence in the vaccine arm was 1.1 cases/1000 PY, vastly lower than in the placebo $\operatorname{arm}(09.1 / 1000 \mathrm{PY})$. VE in protecting against $\mathrm{HZ}$ was nearly the same in the different age groups (•Table 2 ). Here as well, VE was somewhat higher in the modified cohorts than in the ITT group (• Table 2).

In total, 17,531 participants from the ZOE-50 and ZOE-70 studies were includ- 


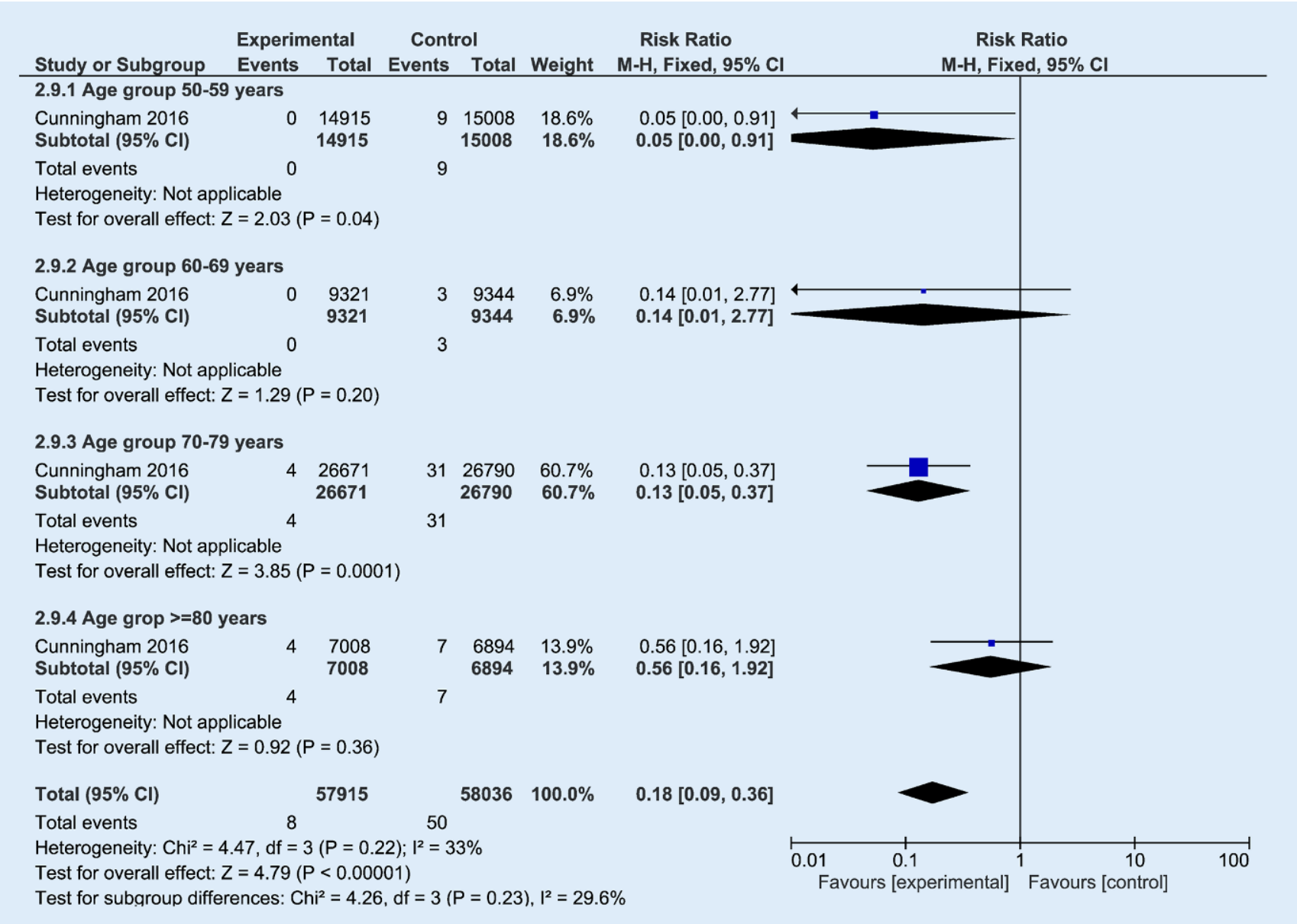

Fig. $3 \Delta$ Efficacy of $\mathrm{HZ} / \mathrm{su}$ inactivated vaccine in preventing postherpetic neuralgia in various age groups $(\geq 50-59, \geq 60-69$, $\geq 70-79$, and $\geq 80$ years); data from the cumulative follow-up periods (total) in person-years [14]

ed in the pooled analysis of participants age $\geq 70$ years. In this population as well, the demographic attributes were similar in the comparison groups.

In the pooled ITT analysis from the ZOE-50 and ZOE-70 studies, VE in protecting against $\mathrm{HZ}$ for adults age $\geq 70$ years was $89.9 \%$ (95\% CI $85.4-93.2 \%$ ); there was no difference between the age groups. In the modified analysis, $\mathrm{VE}$ for adults age $\geq 70$ years was over $91.3 \%$ (95\% CI 86.8 94.5\%) (• Table 2).

Over a period of 3.7 years after vaccination, VE in protecting against $\mathrm{PHN}$ in the pooled ITT analysis was $83.9 \%$ (95\% CI $65.8-93.5 \%$ ) for individuals age $\geq 50$ years and $78.9 \%$ (95\% CI 54.0-91.5\%) for those age $\geq 70$ years. Because of the low number of PHN cases observed in the individual age groups, the confidence intervals for the point estimates in the results of VE were very wide and/or included 1 (- Fig. 3; $\bullet$ Table 2).
7.3 Results of the meta-analysis of data from the ZOE-50 and ZOE-70 studies on the efficacy of the HZ/ su inactivated vaccine in protecting from $\mathrm{HZ}$ and $\mathrm{PHN}$

Data from the ITT groups were analyzed in the meta-analysis of age-specific efficacy against $\mathrm{HZ}$ of the HZ/su vaccine (• Fig. 2). For the age groups $\geq 70$ years, pooled results of the ZOE-50 and ZOE-70 studies were used for the analysis. The meta-analysis showed a VE across all age groups of 92.0\% (95\% CI 89.0-94.0\%). The point estimates of VE declined somewhat with increasing age, from $97 \%$ in participants age 50-59 years to $94 \%$ in those age 60-69 years and $90 \%$ in the age groups 70 years and older; the confidence intervals around the point estimates were overlapped.

Based on the meta-analysis, the efficacy against $\mathrm{PHN}$ was $82.0 \%$ (95\% CI 64.0$91.0 \%$ ) across all age groups. Considering the efficacy in the various age groups, significant vaccine protection was seen only in those 70-79 years old, with $87.0 \%$ (95\% CI 63.0-95.0\%) (• Fig. 3). In the younger age groups and in those over 80 years old, the study populations were too small for this rare event. No clear assessment was possible because of the low number of cases observed. The confidence intervals were wide in all age groups, and some included 1.

\subsection{Duration of protection from $\mathrm{HZ}$ provided by the $\mathrm{HZ} /$ su inactivated vaccine (results of the pooled analysis of the ZOE-50 and ZOE-70 studies)}

Because the incidence of $\mathrm{HZ}$ increases with age, long-term protection provided by the vaccine is especially important. For the duration of vaccine protection against $\mathrm{HZ}$, only data for adults age $\geq 70$ years 


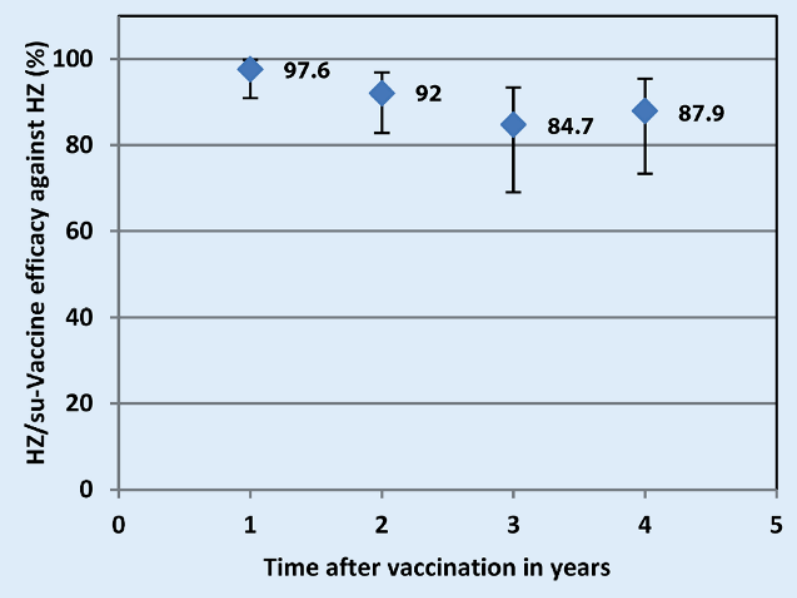

Fig. $4<$ Duration of efficacy of the $\mathrm{HZ} / \mathrm{su}$ inactivated vaccine in preventing $\mathrm{HZ}$ in adults $\geq 70$ years old (ZOE50 + ZOE70) [14]

from the pooled analysis of the ZOE-50 and ZOE-70 studies over a time frame of 4 years were available [14]. The data were from the modified analysis, i.e., participants who did not receive the second vaccine dose or who developed $\mathrm{HZ}$ within 30 days after the second vaccine dose were excluded. Based on the pooled analysis, VE in protecting against $\mathrm{HZ}$ dropped after administration of the second vaccine dose, from $97.6 \%$ (95\% CI 90.9-99.8\%) in the first year to $<90 \%$ from the third year after vaccination (- Fig.4). The data from years 3 and 4 after vaccination suggest that VE reaches a constant plateau as time progresses. A clear interpretation based on clinical data is not possible at this time. No data have been published on the duration of action of the $\mathrm{HZ} / \mathrm{su}$ inactivated vaccine in protecting against $\mathrm{PHN}$.

\subsection{Long-term immunogenicity of the $\mathrm{HZ} / \mathrm{su}$ inactivated vaccine}

Because the duration of vaccine efficacy could be examined for only a short period of time in the RCTs, data from a single-arm phase II multicenter study were referenced in addition to the systematic review. In that study, the immune response of participants age $\geq 60$ years who had received two $\mathrm{HZ} /$ su vaccinations at a 2 -month interval was examined $[16,17]$. A total of 129 participants from Czech Republic, Germany, and the Netherlands were enrolled. The cellular and humoral immune responses induced by vaccination were examined annually over a period of 6 years. The frequency of gE-specif- ic $\mathrm{CD} 4^{+} \mathrm{T}$ cells with at least two expressed activation markers and the geometric mean values of the serum concentration (GMC) of anti-gE antibodies ( $\mathrm{mlU} / \mathrm{mL}$ ) were determined using an ELISA developed by the vaccine manufacturer (cutoff: $18 \mathrm{mIU} / \mathrm{mL}$ ). The subgroup of participants from Czech Republic $(n=68)$ was followed up for a period of 9 years [18]. The median frequency of gE-specific $\mathrm{CD} 4^{+} \mathrm{T}$ cells was highest 3 months after the second vaccine dose $\left(1800 / 10^{6}\right.$ cells). This proportion dropped to $415 / 10^{6}$ cells during the 9 years after vaccination, but at that point it was still more than three times higher than pre-vaccination levels $\left(119 / 10^{6}\right)(\bullet$ Fig. 5). The data indicate that the duration of vaccine-induced protection may be even longer than that confirmed to date in RCTs.

The highest mean concentration of anti-gE antibodies was also measured 3 months after administration of the second vaccine dose $(43,100 \mathrm{mIU} / \mathrm{mL})$, which declined as time progressed. However, in year 9 after the second vaccine dose, it was still seven times higher than pre-vaccination levels (- Fig. 6). From the year 4 after completing the vaccination series, the frequency of gE-specific $\mathrm{CD} 4^{+} \mathrm{T}$ cells and the concentration of anti-gE antibodies remained at constant high levels. This observation fit with the VE findings according to clinical endpoints, which remained at a steady level 3 and 4 years after vaccination.
7.6 Conclusions on the efficacy and effectiveness of the $\mathrm{HZ}$ vaccination

The HZ/su inactivated vaccine can effectively prevent $\mathrm{HZ}$ in people $>50$ years; efficacy is $92 \%$ across all age groups. Protection against $\mathrm{HZ}$ falls slightly with increasing age but remains over $90 \%$ in adults 70 years and over. Thus, the HZ/ su inactivated vaccine confers a high level of protection at all ages and also in older people with the highest risk of HZ. This is an advantage compared with the live attenuated vaccine, which has a markedly lower VE in older age groups. Protection drops from $98 \%$ in the first year to $85 \%$ in the third year after vaccination and remains at $88 \%$ in the fourth year after vaccination. Further conclusions on the duration of protection for the clinical endpoint $\mathrm{HZ}$ are not yet possible. Based on immunological data available for a period of 9 years after vaccination, the immune response remains at a level that is multiple times higher than before vaccination. This might indicate a prolonged duration of vaccine-induced protection. The HZ/su inactivated vaccine can prevent the occurrence of PHN. This effect is derived from the effective prevention of $\mathrm{HZ}$ as a precondition for the prevention of its sequelae. Because of the rarity of this event, significant results on protection against $\mathrm{PHN}$ are available only for the entire cohort, and age-related results are available only for the largest cohort recruited, participants age $70-79$ years. Overall, VE is $82 \%$, and it is $87 \%$ among 70 - to 79 -year-olds.

\section{Vaccine reactogenicity and safety}

\subsection{Approach and studies considered}

The STIKO working group assessed severe adverse drug reactions ( 8 points) and severe pain at the point of injection (7 points) as essential endpoints. Fever as a systemic reaction was classified as an important endpoint (5 points); swelling and other local reactions were considered less important endpoints (3 points) (- Table 1).

Results from three RCTs that recorded the abovementioned endpoints were 

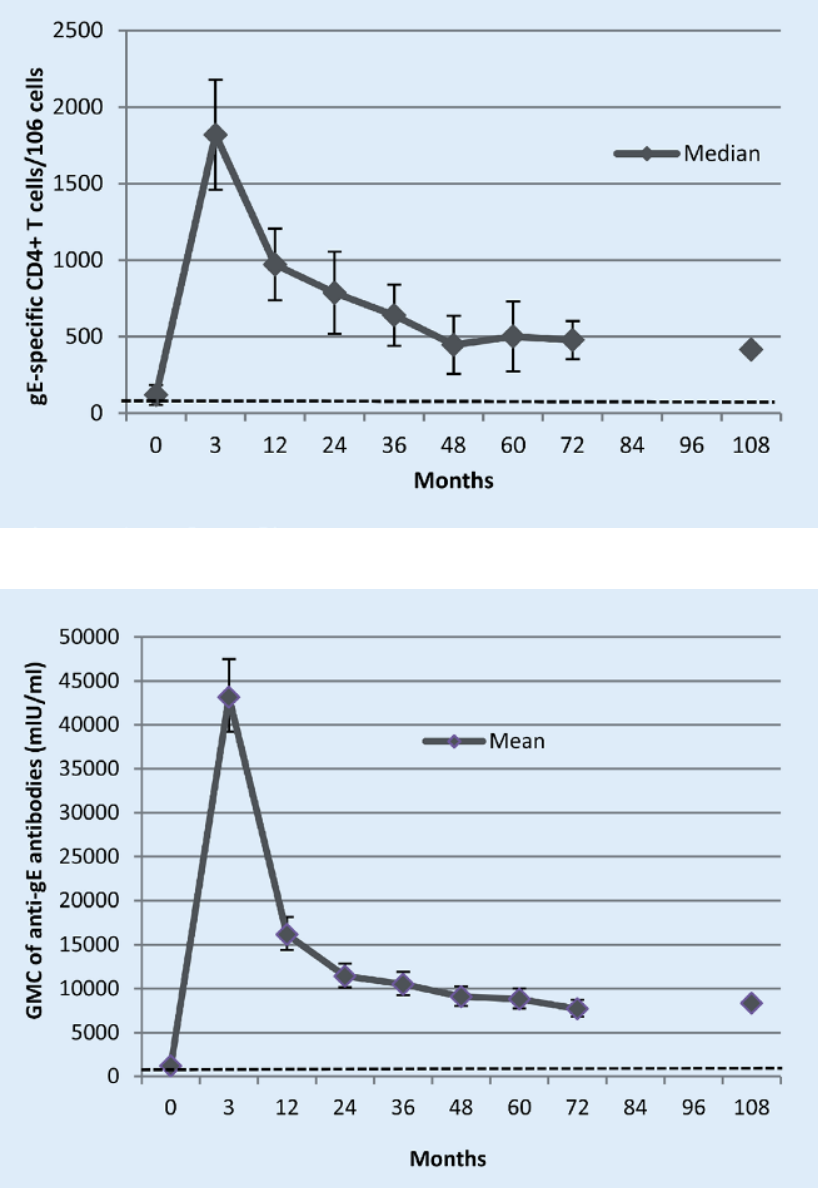

Fig. $5<$ Median and $95 \%$ confidence intervals of the frequency of VZV glycoprotein $\mathrm{E}$ (gE)-specific CD4+ $T$ cells over 108 months [16-18]

Fig. $6<$ Geometric mean values of serum concentration (GMC) of anti-gE antibodies ( $\mathrm{mlU} / \mathrm{mL}$ ) with $95 \%$ confidence intervals over 108 months [16-18]

included in the safety evaluation. These RCTs were the ZOE-50 [15] and ZOE-70 studies [14], described in subsections 7.1 and 7.2 above, and results from two study arms of a phase II study [19]. The included RCTs had a low risk of bias. Details on the studies and results of the aggregate evaluation are presented hereinafter.

One subgroup of participants from the ZOE-50 study was asked to document local reactions at the site of injection (pain, redness, and swelling) and systemic reactions (fatigue, fever, headache, and myalgia) in a diary for 7 days [15]. This subgroup included all participants age $\geq 70$ years and selected randomized participants from younger age groups. Redness and swelling at the injection site were assessed using diameter on a scale from 0 $(<20 \mathrm{~mm})$ to $3(>100 \mathrm{~mm})$. Fever, preferably measured orally, was also assessed using a 4-point scale from $0\left(<37.5^{\circ} \mathrm{C}\right)$ to 3 $\left(>39.0^{\circ} \mathrm{C}\right)$. For other side effects intensity was classified on a scale from 0 (none) to 3 (common everyday activities are im- possible). Other adverse side effects were registered as spontaneous reports for a period of 30 days after every vaccine dose. Other severe side effects were measured for a period of at least 12 months after administration of the second dose. All health complaints with any link to the study, all deaths, and all potentially immune-mediated discomfort were evaluated for the entire study duration of 3.5 years. A total of 8926 participants were included in the subgroup for safety evaluation of the $\mathrm{HZ} /$ su inactivated vaccine (4460 from the vaccine and 4466 from the placebo arms).

In the ZOE-70 study, a random sample group of 1025 participants $(7.4 \%$ of the total study population; 512 from the vaccine arm and 513 from the placebo arm) was recruited from among the study participants in the safety evaluation [14]. The evaluation procedure was identical to that of the ZOE-50 study; the study duration was 4 years.

In a multi-arm, phase II multicenter study, the safety of the HZ/su inactivat- ed vaccine in various doses was tested among participants age $\geq 50$ years [19]. The study included a total of 410 participants from Czech Republic, Spain, and the United States (US). Data from the placebo $\operatorname{arm}(n=38)$ and the arm with a later vaccine concentration $(n=150)$ were extracted. The safety evaluation was identical to that of the ZOE-50 and ZOE-70 studies. Every major event over a time frame of 14 months was evaluated.

\subsection{Local reactions after the $\mathrm{HZ} / \mathrm{su}$ inactivated vaccine}

In clinical studies of the safety of the $\mathrm{HZ} / \mathrm{su}$ inactivated vaccine, participants in the vaccine arm reported local reactions at the site of injection significantly more frequently than participants in the placebo arm $(81 \%$ vs. $12 \%)$. Pain was the most frequent local reaction. Local reactions of the highest intensity (grade 3 ) occurred in $9.4 \%$ versus $0.3 \%$ of participants (• Fig. 7 ). The frequency of vaccine reactions was not significantly increased after administration of the second vaccine dose [14]. Vaccine reactions were independent of age; $53 \%$ of participants age $\geq 80$ years and $55 \%$ of those age 70-79 years reported vaccine reactions [14]. All reactions were temporary and lasted a median of 2-3 days.

\subsection{Systemic reactions after the $\mathrm{HZ} /$ su inactivated vaccine}

Systemic side effects associated with the vaccine were also more frequent in the intervention arm $(65 \%)$ than in the placebo arm (29\%). Systemic reactions of the highest intensity (grade 3 ) occurred in $10.6 \%$ of participants in the vaccine arm and $2.4 \%$ of those in the placebo arm (- Fig. 8). The frequency of the systemic reactions fever, fatigue, myalgia, and headache were described in the ZOE-70 study, each with regard to intensity and for grade 3 (see the appendix).

\subsection{Severe adverse events caused by the vaccine (SAE)}

In the three clinical trials examining the safety of the HZ/su inactivated vaccine, 24 of 29,499 participants experienced SAEs in connection with the vaccine, of which 


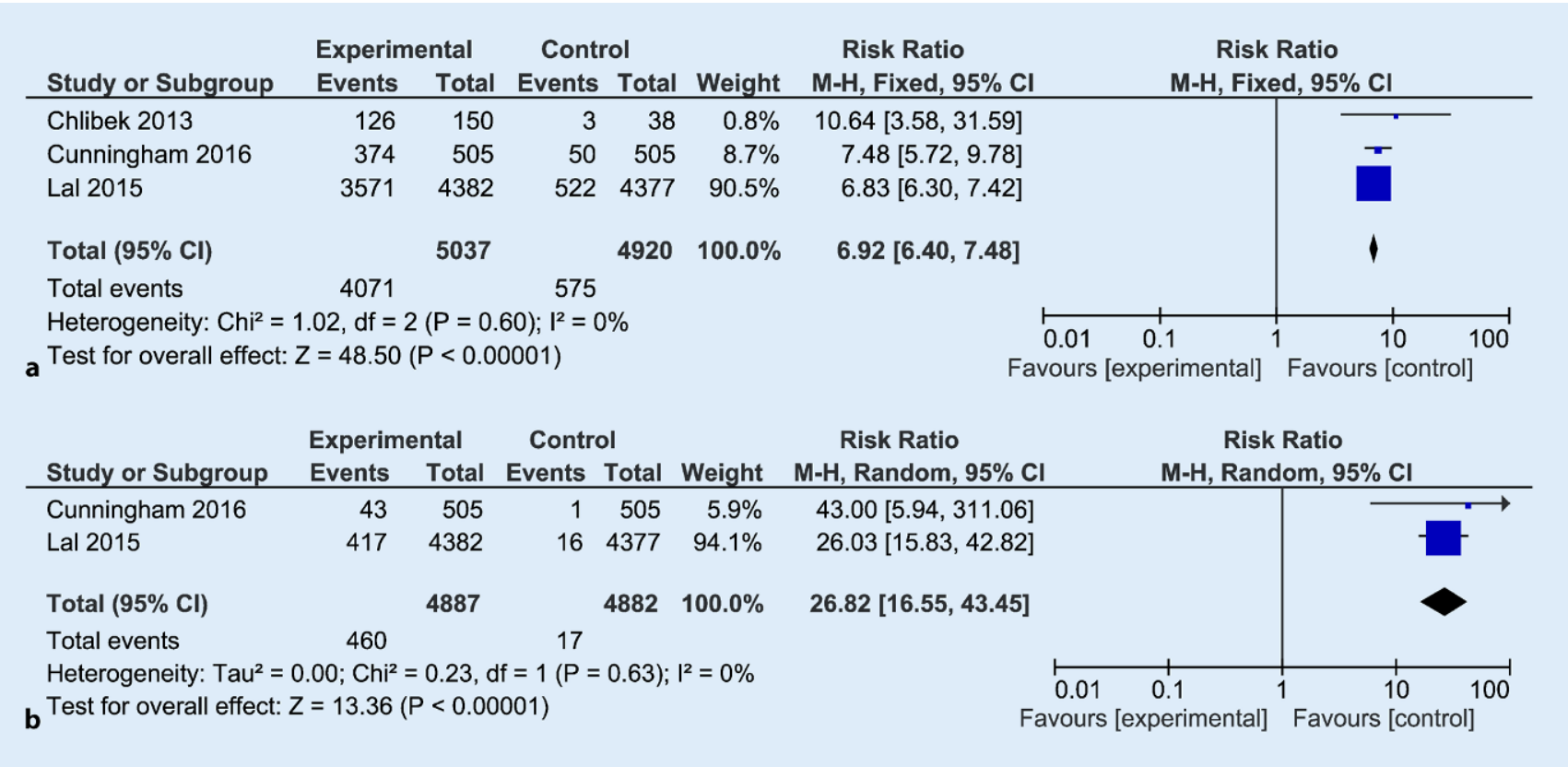

Fig. $7 \Delta$ Forest plots of relative risks (RR) of local reactions to the HZ/su inactivated vaccine (all degrees and grade 3). a Local reactions at the injection site (all degrees), $\mathbf{b}$ Local reactions at the injection site (grade 3 )

13 occurred in the vaccine arm and 11 in the placebo arm (- Fig. 8). The following syndromes were recorded as SAEs in the vaccine arm: hypotension with syncope, lymphadenitis, myocardial infarction, ulcerative colitis, pancreatitis, erythema and pain at the injection site, shivering, fever, allergic granulomatous vasculitis, bacterial joint inflammation, erysipelas, HZ oticus, eczema, neutropenic sepsis, and acute myeloid leukemia (for details, see appendix).

SAEs that were considered by the responsible reviewers to be related to intervention, potentially immune-mediated diseases, and deaths occurred with comparable frequency in the study arms (vaccine and placebo arm) of the ZOE-50 and ZOE-70 studies (• Fig. 9).

The reviewers initially classified one death in the HZ/su arm of the ZOE-70 study as associated with the vaccine. This case occurred in a 90 -year-old participant with preexisting thrombocytopenia who was diagnosed with acute myeloid leukemia (AML) 75 days after the first dose of $\mathrm{HZ} / \mathrm{su}$ and died from neutropenic sepsis 97 days after vaccination, without having received the second dose. The CHMP (Committee for Medicinal Products for Human Use) considered a relationship between vaccination and AML to be highly unlikely as the neutropenic sepsis and subsequent events were considered most likely side effects of ongoing therapy with azacitidine [20].

\subsection{Conclusions on the safety of the $\mathrm{HZ} /$ su inactivated vaccine}

The HZ/su inactivated vaccine is exceptionally reactogenic. Local reactions and grade 3 systemic reactions occurred in roughly every 10th vaccinated person. However, the vaccine reactions do not last long ( 1 to 2 days for reactions of the highest degree). In marketing authorization studies, there were no warnings about severe side effects or potentially immune-mediated diseases. The frequency of SAEs was the same in the vaccine group and in the placebo group. SAEs considered to be vaccine related mainly included health conditions or diseases that are generally not rare in the investigated age groups. One fatal case was initially regarded as vaccine related, but the CHMP deemed a causal relationship to be unlikely.

\section{Evidence profile of the efficacy and safety of the $\mathrm{HZ} / \mathrm{su}$ inactivated vaccine}

To assess the quality of available evidence for the efficacy and safety of the HZ/su in- activated vaccine, an evidence profile was drawn up of pre-defined PICO questions using GRADEprofiler software. Relevant effect estimates observed for each endpoint and the quality of evidence for these estimators have been compiled in this profile (• Table 3).

The quality of evidence for efficacy of the $\mathrm{HZ} /$ su inactivated vaccine in preventing $\mathrm{HZ}$ is classified as high; the quality of evidence for efficacy against PHN is classified as low, and the quality of evidence for the safety of the vaccine is estimated as moderate.

\section{Implementing the $\mathrm{HZ} / \mathrm{su}$ vaccination}

\subsection{Dose, type, and duration of administration}

The $\mathrm{HZ} / \mathrm{su}$ vaccine is approved for use in adults age 50 years and older. The vaccination scheme is two i.m. vaccinations at least 2 months apart. In addition to the vaccination interval of 2 months, immunogenicity studies have also examined vaccination intervals of 6 and 12 months and determined the vaccine response rates 1 month after administering the second dose in each case [21]. It was shown that the immune response to vaccination on a 


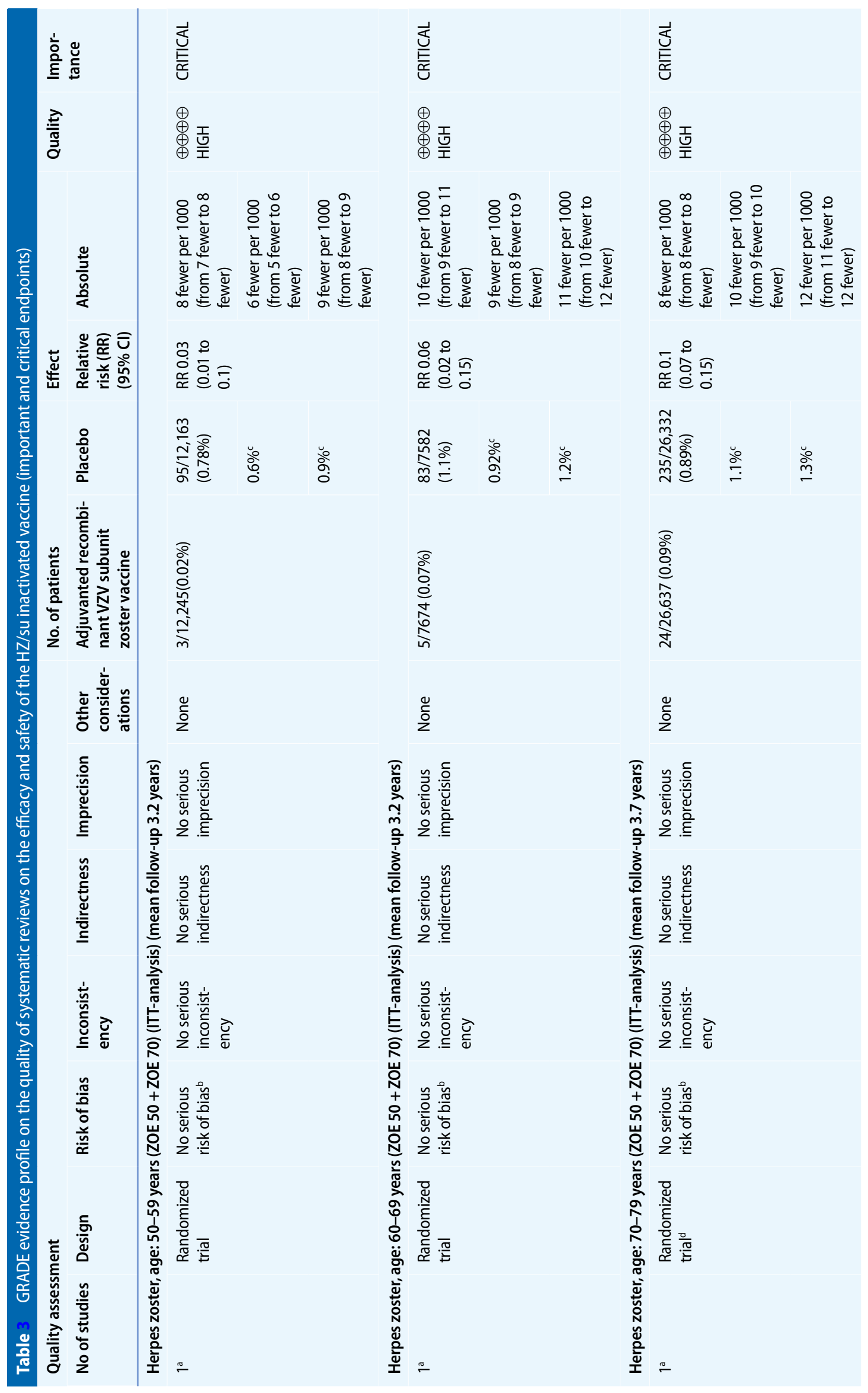




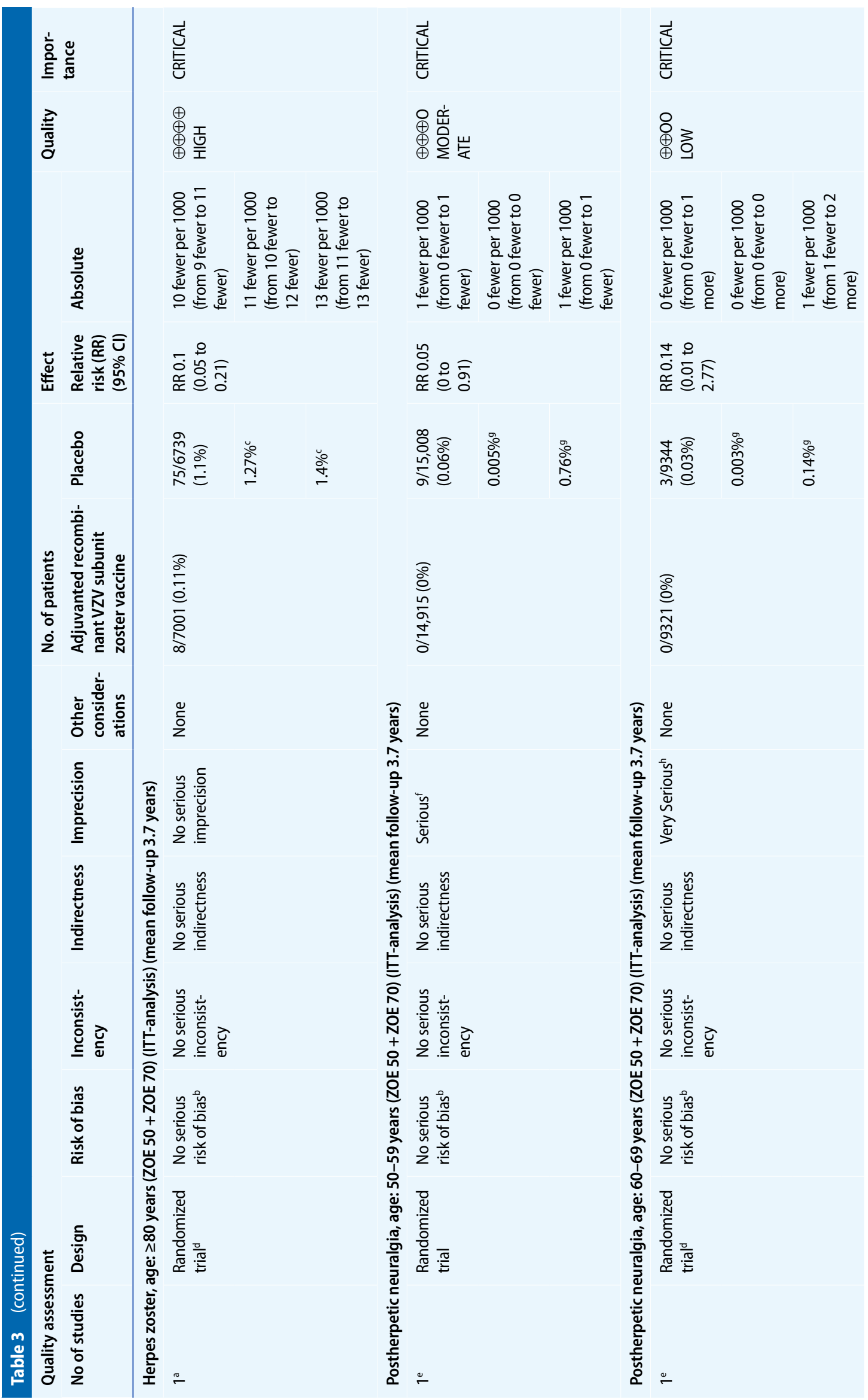




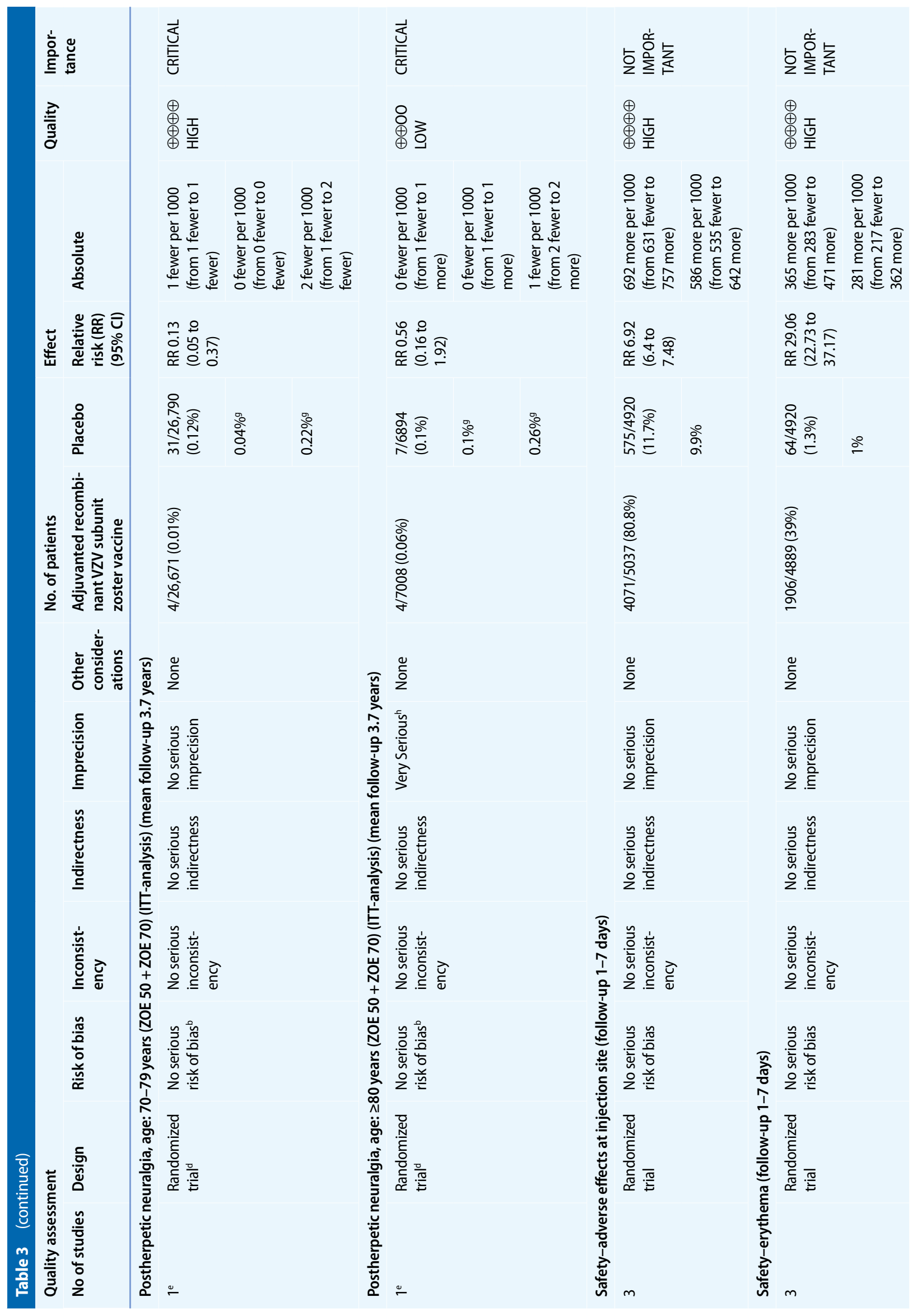




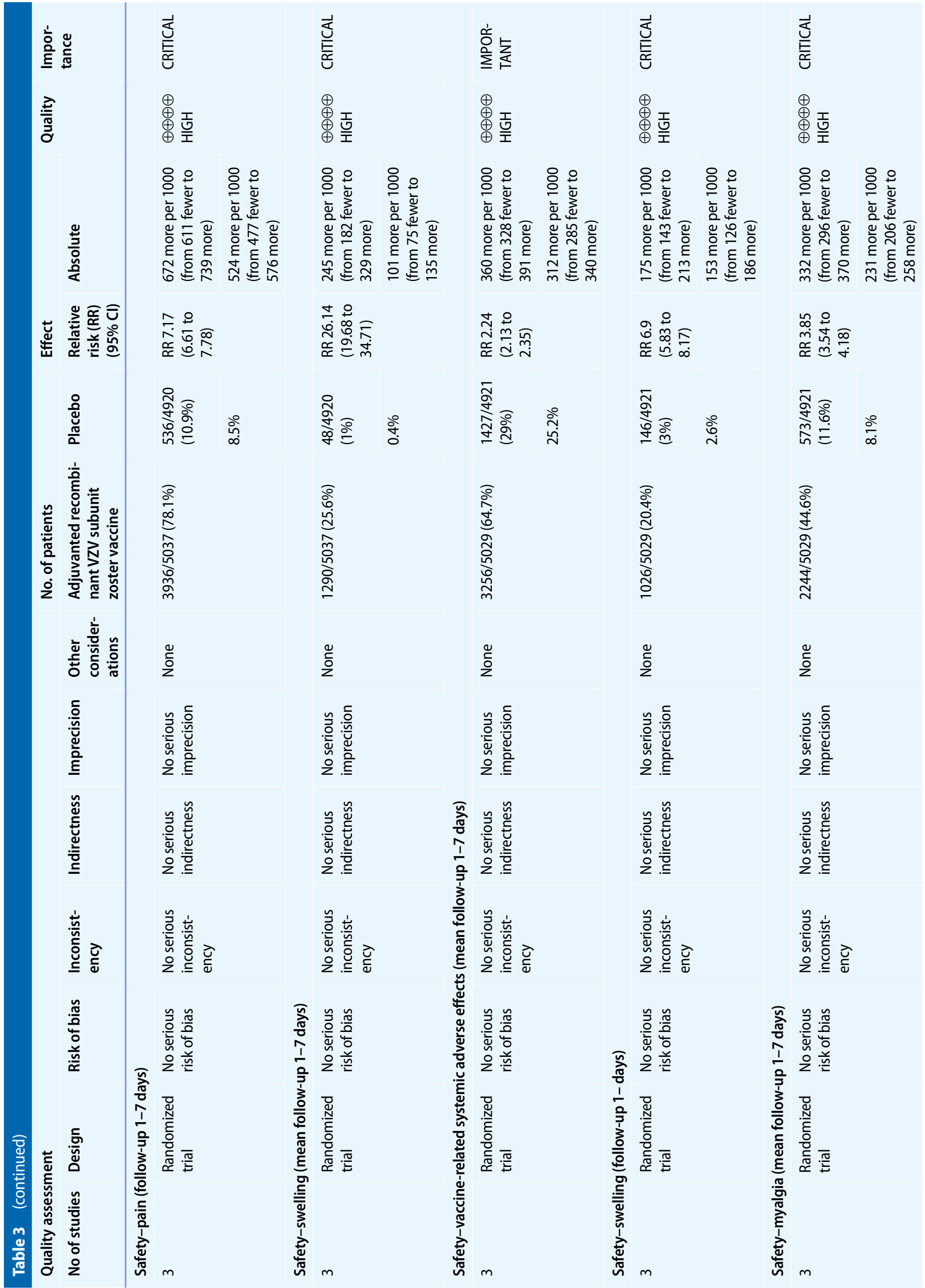




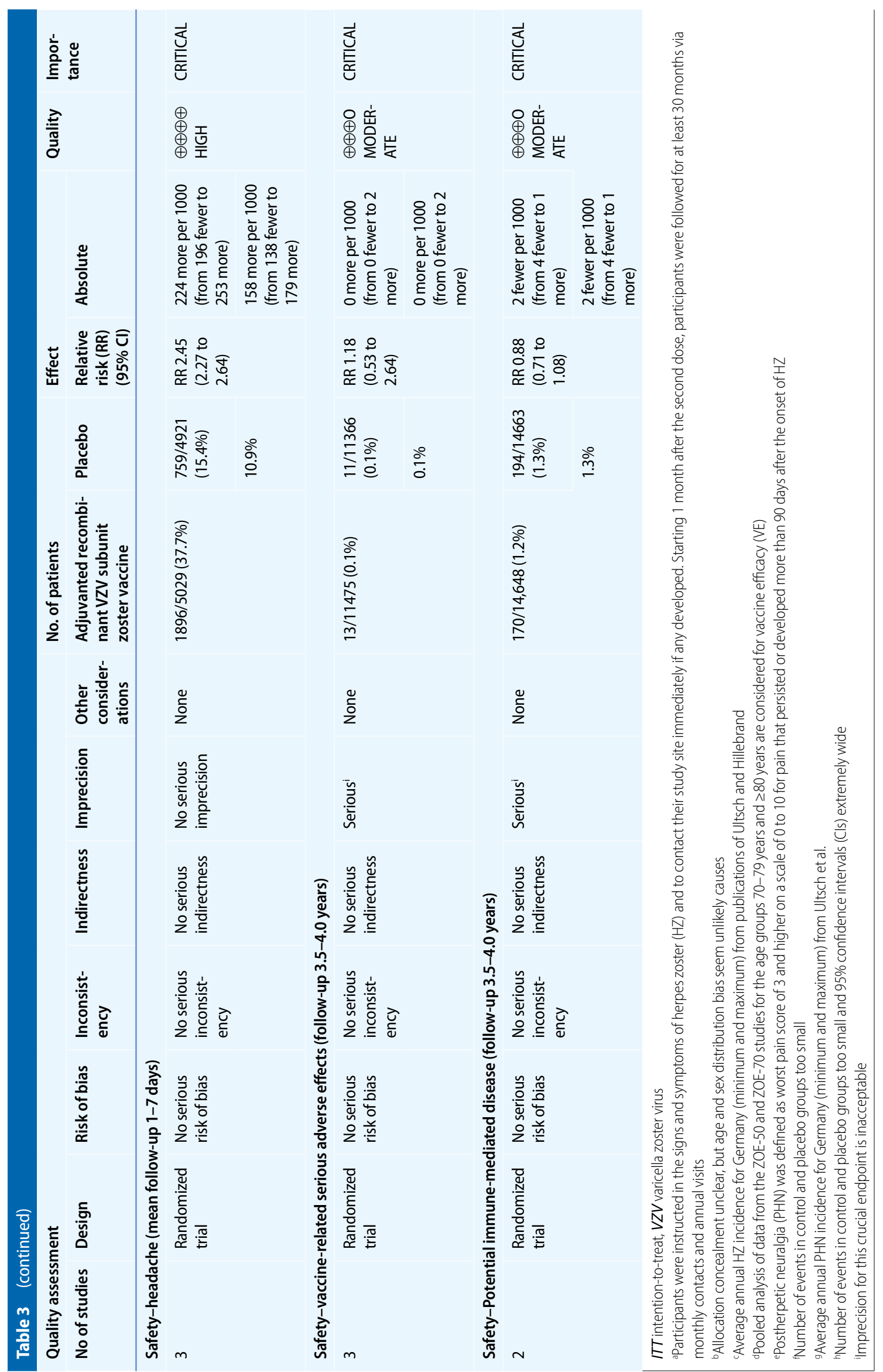




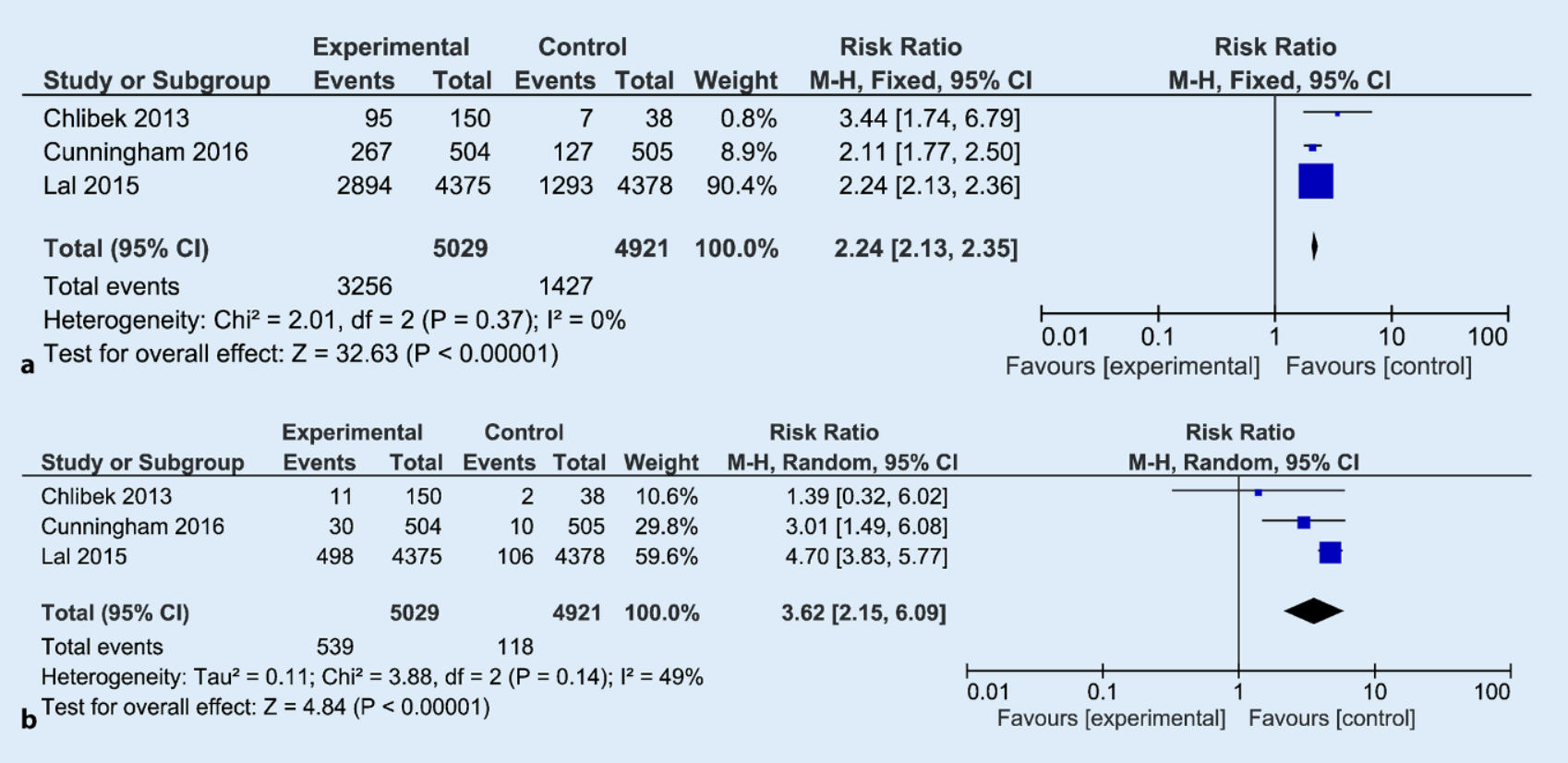

Fig. $8 \Delta$ Forest plots of the relative risks (RR) of adverse systemic side effects to the HZ/su inactivated vaccine (all degrees and grade 3). a Systemic adverse side effects (all degrees), b Systemic adverse side effects (grade 3 )

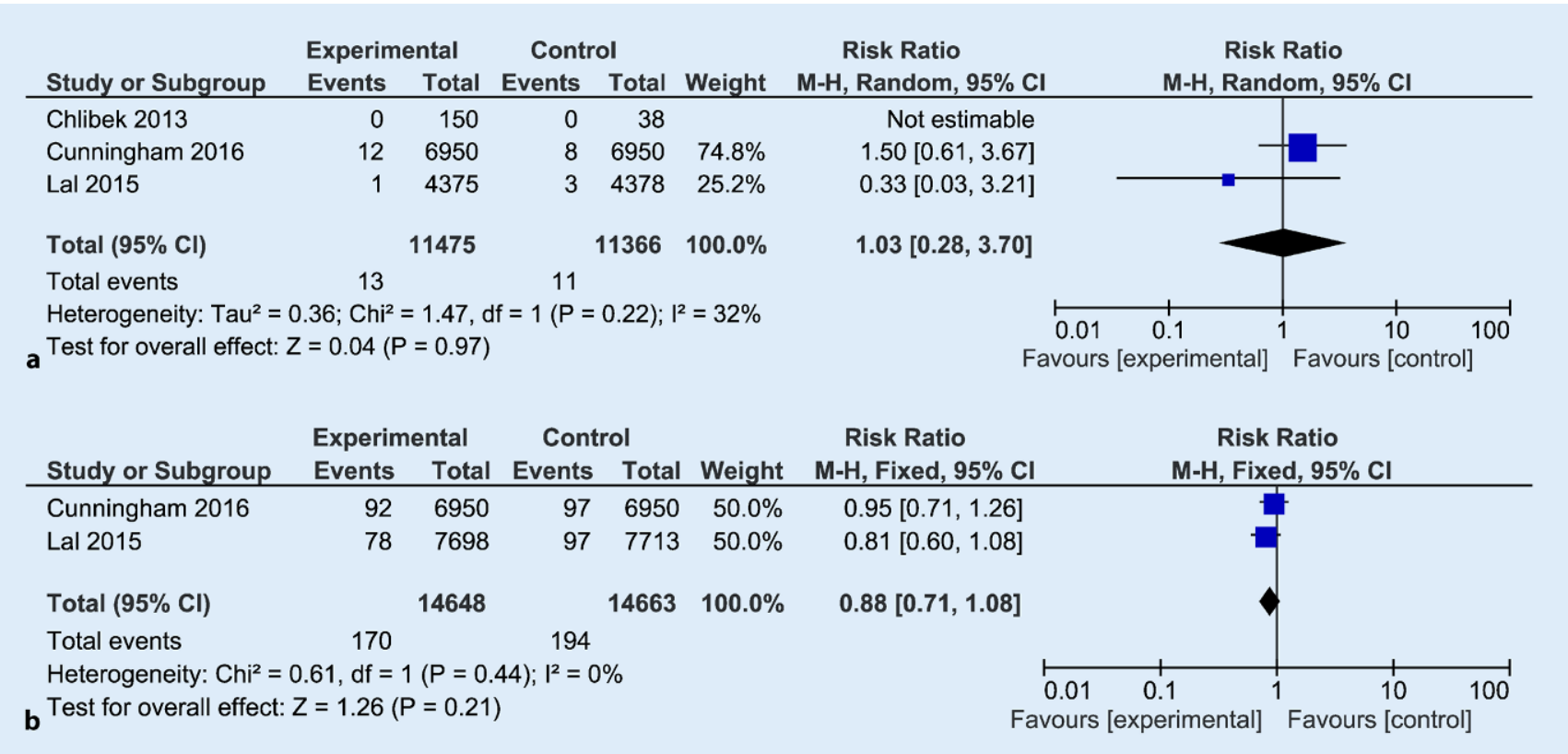

Fig. $9 \Delta$ Forest plots of the relative risks (RR) for severe adverse effects of the $\mathrm{HZ} /$ su inactivated vaccine and possible immune-mediated diseases. a Possible vaccination-related severe adverse effects, $\mathbf{b}$ Possible immune-mediated diseases

0-6 month schedule was the only one not inferior to the $0-2$ month schedule. Based on these findings, administration of the second vaccine dose can be delayed for up to 6 months without concern about a loss in the target immune response. If administration of the second vaccine dose is delayed for more than 6 months, the immune response appears to be somewhat reduced, but study data show that anti-gE antibody concentrations are more than 11 times higher than pre-vaccination levels. No study data are available regarding the need for booster doses or repeated vaccinations. Results from long-term observations on the duration of protection after vaccination are required, ideally with the clinical endpoint of $\mathrm{HZ}$.

\subsection{Coadministration with other vaccines}

The immunogenicity and safety of the HZ/ su inactivated vaccine with simultaneous administration of a quadrivalent seasonal influenza vaccine (QIV) were studied in an open, randomized phase III trial in adults age $\geq 50$ years. Participants $(n=828)$ 
were allocated to one of two groups at a ratio of 1:1. Participants received the first $\mathrm{HZ} / \mathrm{su}$ inactivated vaccine dose together with the QIV dose, followed by a second $\mathrm{HZ} / \mathrm{su}$ dose 2 months later (intervention arm), or the QIV first and the two HZ/su inactivated vaccine doses 2 and 4 months later (control arm). According to measured anti-gE concentrations (cut-off: 97 $\mathrm{mIU} / \mathrm{mL}$ ), a similar number of participants in the intervention arm $(95.8 \%$, 95\% CI 93.3-97.6\%) and the control arm (97.9\%, 95\% CI 96.0-99.1\%) responded to the $\mathrm{HZ} / \mathrm{su}$ inactivated vaccine. At the same time, it could be demonstrated that the antibody concentrations to the HZ/ su and QIV vaccines were not inferior to those in the control arm. Safety concerns regarding simultaneous administration were not observed [22]. Simultaneous administration of the $\mathrm{HZ} / \mathrm{su}$ inactivated vaccine and a non-adjuvanted, inactivated, seasonal influenza vaccine on different limbs is possible, according to the product information.

Simultaneous administration of the $\mathrm{HZ} /$ su inactivated vaccine with the 23-valent pneumococcal polysaccharide vaccine (PPSV23) was studied in 865 adult participants age 50 years and older (average age 63.2 years) in an open randomized trial [23]. Here as well, participants were assigned to the coadministration or control arm at a ratio of $1: 1$, and the vaccination scheme in both arms corresponded to that of the study on the simultaneous administration of QIV. In total, 98\% of participants in both arms responded to the $\mathrm{HZ} / \mathrm{su}$ inactivated vaccine, and similar antibody concentrations were measured. No safety concerns arose in this study either. The study results were not taken into account in the current valid version of the product information (last updated in March 2018), and coadministration is not yet covered by marketing authorization.

\section{$10.3 \mathrm{HZ} /$ su vaccination after prior $\mathrm{HZ}$ disease}

The immunogenicity and safety of the $\mathrm{HZ} /$ su inactivated vaccine in patients $\geq 50$ years after prior $\mathrm{HZ}$ disease were studied in a non-randomized, open, multicenter trial in Canada and Russia between June
2013 and November 2014 [24]. Among participants in the study $(n=96), 68 \%$ $(n=65)$ had developed HZ within the past 4 years and none had ongoing active symptoms; in 19\% ( $n=18)$ of participants, the HZ episode occurred 5-9 years earlier and in $14 \%(n=13)$, it was 10 or more years earlier. The median participant age was 64 years (range: $50-89$ years). Participants received two doses of $\mathrm{HZ} / \mathrm{su}$ at an interval of 2 months and were observed for a follow-up period of 12 months. Immunogenicity of the vaccine was measured as the rise in titer of anti-gE antibodies 1 month after the second vaccination. The primary goal of the study was a fourfold increase in titer over baseline before vaccination; this was achieved in $90.2 \%$ (95\% CI 81.7-95.7\%) of participants. Local and systemic side effects were documented for 7 days and adverse events for 30 days after each vaccination. Severe side effects occurring at any time during the study were recorded. The results confirmed the findings of the marketing authorization studies. With nine HZ episodes reported by six participants over a period of 12 months after vaccination, more recurrent episodes occurred than expected based on observational studies in unvaccinated people with anamnestic HZ. However, the HZ diagnoses were not confirmed by laboratory investigation and three patients had not consulted a physician. In summary, it can be stated that the HZ/su inactivated vaccine disease is sufficiently immunogenic in adults age $\geq 50$ years with prior $\mathrm{HZ}$, and no safety concerns have been identified.

\section{$10.4 \mathrm{HZ} /$ su vaccination after prior $\mathrm{HZ}$ live vaccine}

An open, multicenter phase III study was conducted to investigate whether the immune response to the $\mathrm{HZ} / \mathrm{su}$ inactivated vaccine in adults age $\geq 65$ years who had been vaccinated with the live $\mathrm{HZ}$ vaccine 5 or more years previously is comparable to that in previously naive individuals [25]. The study was conducted in the US between March and August 2016, with 430 study participants allocated to one of the two groups at a ratio of 1:1. In parallel, the tolerability of $\mathrm{HZ} / \mathrm{su}$ vaccination after prior $\mathrm{HZ}$ live vaccine was studied; safety monitoring was conducted through August 2017. The aim of the study was to compare the humoral immune response to the two vaccination regimens 1 month after the second vaccine dose using the GMC of anti-gE antibodies, and to demonstrate non-inferiority of the previously vaccinated group to the naive group. Among participants previously vaccinated with the live vaccine, the humoral immune response to the HZ/ su inactivated vaccine was not worse than the response among those not previously vaccinated. There was also no difference between the groups in cellular immunogenicity, reactogenicity, or safety [25]. Based on these findings, there are no objections to use of the $\mathrm{HZ} / \mathrm{su}$ inactivated vaccine in people previously vaccinated with the live vaccine, as long as there is an interval of at least 5 years between the two vaccinations.

\section{$10.5 \mathrm{HZ} /$ su vaccination in patients with bleeding tendency}

The HZ/su inactivated vaccine is approved for i. m. injection. In an open, randomized phase III trial (participant ratio 1:1), the safety and immunogenicity of subcutaneous (s.c.) injection were compared to that of i.m. injection in two vaccinations (at 0 and 2 months) [26]. A total of 60 participants from Japan age $\geq 50$ years were included. The age distribution was identical in the two groups, with mean age 61.9 years; $50 \%$ of participants were female. Anticipated and unanticipated reactions after administration of the vaccine were documented for 7 and 30 days after vaccination, and their severity was assessed according to grades 1 to 3 . Although the immune response after vaccination was equally high in both groups, the reactogenicity of the vaccine in the s.c.-vaccinated group was markedly stronger than that in the i.m. group. According to the product information, the $\mathrm{HZ} / \mathrm{su}$ inactivated vaccine is approved only for i.m. administration. 
10.6 HZ/su vaccination in patients with immunosuppression and other underlying diseases

\subsubsection{Patients after autologous stem-cell transplantation}

In the first year after hematopoietic stemcell transplantation (HSCT), the risk of developing HZ is markedly higher [27] and $\mathrm{HZ}$ disease can be complicated by visceral dissemination [28]. The safety and immunogenicity of the HZ/su inactivated vaccine were studied in an observer-blinded, placebo-controlled phase 1/2a trial between 2009 and 2012 in the US among individuals who had received an autologous stem-cell transplant 50-70 days previously [29]. The 121 study participants were randomized at a ratio of 1:1:1:1 and received either three doses $\mathrm{gE} / \mathrm{ASO}_{\mathrm{B}}$ (later vaccine), or three doses $\mathrm{gE} / \mathrm{ASOl}_{\mathrm{E}}$, or one dose physiological saline solution and two doses $\mathrm{gE} / \mathrm{ASO}_{\mathrm{B}}$, or three doses physiological saline solution, at months 0,1 , and 3. Regardless of the vaccine formula, the gE-specific $\mathrm{CD} 4^{+}$cell counts and anti-gE serum antibody titers 1 month after the last vaccine dose were both higher than those in the placebo arm, and titer levels remained constantly high for 1 year. Both vaccine formulas were well tolerated and triggered a satisfactory immune response that remained for a period of 1 year.

The efficacy and safety of the HZ/su inactivated vaccine in patients after autologous HSCT were also studied for 21 months in a randomized, observer-blinded, placebo-controlled phase 3 trial stratified into two age groups (18-49 years and $\geq 50$ years) [30]. In total, 1721 (93.2\%) of the 1846 participants vaccinated after HSCT were included in the analysis. The efficacy against $\mathrm{HZ}$ was $68 \%$, and $89 \%$ against PHN; this was the same for both age groups. There were no safety concerns.

$\mathrm{HZ}$ and PHN can be effectively prevented with the $\mathrm{HZ} / \mathrm{su}$ inactivated vaccine in patients after autologous HSCT, regardless of age.

\subsubsection{Persons with HIV}

People with HIV have a markedly higher risk of developing $\mathrm{HZ}$ [31, 32]. Antiretroviral therapy (ART) clearly reduces the risk of HZ, but it is still 3-5 times high- er than in people with healthy immune systems [33]. The safety and immunogenicity of the HZ/su inactivated vaccine were studied in an observer-blinded, placebo-controlled phase $1 / 2 \mathrm{a}$ trial in Germany, the US, and the UK between September 2010 and May 2013 [34]. The following three groups of patients with HIV were studied; participants differed with regard to immune status $\left(\mathrm{CD} 4^{+}\right.$cell count) and ART: (i) ART and $\mathrm{CD} 4^{+}$cell count $\geq 200$ cells $/ \mathrm{mm}^{3}(n=95)$; (ii) ART and $\mathrm{CD}^{+}$cell count $<200$ cells $/ \mathrm{mm}^{3}$ ( $n=14$ ), (iii) no ART and CD4 $4^{+}$cell count $\geq 500$ cells $/ \mathrm{mm}^{3}(n=15)$. The groups were randomized at a ratio of 3:2 and received the $\mathrm{HZ} / \mathrm{su}$ inactivated vaccine or saline solution at months 0,2 , and 6 . Of the 123 enrolled participants (average age 46 years), 112 (91.1\%) completed the study. Local and systemic reactions occurred in the vaccine arm more frequently than in the placebo arm, but generally did not last long (median: 1-3 days) and were mild to moderate in intensity. Up to $16.4 \%$ of participants in the vaccine arm and $8.3 \%$ of those in the placebo arm complained of severe (grade 3 ) local or general reactions. SAEs were not observed. Over a follow-up period of 18 months, the vaccine showed no sustained negative effects on HIV viral load or immune status (measured by $\mathrm{CD} 4^{+}$cell count). One month after the third vaccine dose, the gE-specific cell-mediated immune response and anti-gE serum antibody titer were significantly higher in the verum group than in the placebo group, and remained higher than pre-vaccination titers for a period of 12 months. Based on the study results, the HZ/su vaccine is sufficiently immunogenic in people with HIV and has an acceptable safety profile. Because the gE-specific, cell-mediated immune response barely increases after the third vaccine dose, a two-dose scheme is deemed sufficient for successful vaccination.

\subsubsection{Patients with tumors}

Patients with solid tumors who are receiving immunosuppressive therapy have a fourfold greater risk of developing HZ. In a randomized, observer-blinded, placebo-controlled phase $2 / 3$ study, the immunogenicity and safety of the $\mathrm{HZ} /$ su inacti- vated vaccine were studied in patients 18 years and over $(n=347)$ receiving immunosuppressive therapy for a tumor disease in Canada, Czech Republic, Korea, France, the UK, and Spain [35]. Participants in the vaccine and placebo arms (1:1) were allocated into two further subgroups (each at a ratio of 4:1) who received two vaccine doses i.m. at an interval of 1-2 months. The first dose was administered either 8-30 days before starting the chemotherapy cycle (group A, pre-chemo) or at the start of the chemotherapy cycle (group $\mathrm{B}$, on chemo). One month after the second vaccine dose, the vaccine induced a robust immune response regardless of whether vaccination started before or at the same time as the chemotherapy cycle. The immune response persisted for up to 12 months after the second dose. The vaccine was well tolerated, and there were no safety concerns.

In another 1:1 randomized, placebo-controlled, observer-blinded phase 3 trial, the safety and immunogenicity of the $\mathrm{HZ} /$ su inactivated vaccine were studied in patients $\geq 18$ years $(n=562)$ with tumors of the lymphatic system (multiple myeloma, Hodgkin lymphoma, chronic lymphocytic leukemia, non-Hodgkin lymphoma) [36]. Participants received two doses at an interval of 1-2 months $\geq 10$ days before or after chemotherapy. A total of 562 participants were included (HZ/su group: 283; placebo group: 279). The preliminary results showed that vaccination induces robust humoral (anti-gE antibodies) and cellular (gE-specific $\mathrm{CD} 4^{+} \mathrm{T}$ cells) immunity. No safety concerns arose up to 6 months after administration of the second dose in an ongoing study with blinding maintained.

\subsubsection{Patients after kidney transplant}

As a result of lifelong immunosuppressive therapy, people who have received a kidney transplant have a sevenfold higher risk of developing HZ. In a randomized, double-blind, placebo-controlled phase 3 trial, the immunogenicity and safety of the $\mathrm{HZ} /$ su inactivated vaccine were studied in individuals who had received a kidney transplant in Belgium, Canada, Czech Republic, Finland, Italy, Panama, Korea, Spain, and Taiwan 
in two different age cohorts (18-49 years and 50+ years) [37]. Participants ( $n=123$ in the vaccine arm and $n=132$ in the placebo arm) received two vaccinations $i . m$. at an interval of 1-2 months, administered 4-8 months after transplantation. Preliminary data showed that the HZ/ su inactivated vaccine induces a robust humoral and cell-mediated immune response up to 1 month after the second vaccination. The humoral immune response was higher in the younger age cohort than in the cohort age 50 years and over. There were no safety concerns up to 1 month after administration of the second dose.

\subsubsection{Summary of $\mathrm{HZ} / \mathrm{su}$ vaccination in patients with immunosuppression and underlying diseases}

Depending on their underlying disease and/or therapy, patients with immunosuppression have a markedly increased risk of $\mathrm{HZ}$ and subsequent $\mathrm{PHN}$. In vaccine studies, the efficacy, immunogenicity, and safety of the $\mathrm{HZ} / \mathrm{su}$ inactivated vaccine have been examined in the following risk groups: patients after HSCT, people infected with HIV, kidney transplant recipients, patients with tumors of the lymphatic system before or after immunosuppressive chemotherapy, and patients with solid tumors before or after immunosuppressive chemotherapy. In patients after HSCT, the HZ/su inactivated vaccine demonstrated efficacy of $64-69 \%$ in protecting against $\mathrm{HZ}$ and 84 $90 \%$ in protecting against $\mathrm{PHN}$. In other patient groups, vaccination induced robust humoral and cellular immune responses that lasted more than 12 months. The vaccine is reactogenic, generally well tolerated, and there is no evidence of SAEs in this patient group. According to the product information, the vaccine is not contraindicated for patients with immunosuppression. It must be noted that, as with other vaccines, it is possible that these patients may not achieve a sufficient immune response. An individual risk-benefit assessment before vaccination is advised [9].

In light of the examined efficacy and safety of the $\mathrm{HZ} /$ su inactivated vaccine in particularly vulnerable groups of pa- tients with an impaired immune system, it can be assumed that the vaccine is safe and effective for people with chronic underlying diseases who also have an increased risk of $\mathrm{HZ}$ disease and complications. This is supported by the results of a post-hoc analysis using data of the pooled ZOE-50 and ZOE-70 trials conducted in patients with underlying diseases such as arthritis, chronic renal disease, chronic obstructive pulmonary disease, coronary disease, and diabetes mellitus. In these patients, the analysis showed an efficacy level of the vaccine that corresponds to the overall efficacy against $\operatorname{HZ}[9,20]$.

\section{Acceptance and feasibility of the $\mathrm{HZ} /$ su vaccination}

Acceptance of a vaccine in the target population is an important prerequisite for the successful implementation of a new vaccination recommendation. For an individual decision on $\mathrm{HZ}$ vaccination, it is necessary to be aware of $\mathrm{HZ}$ disease, to be able to estimate the severity of the disease, and to know that there is a vaccine that can provide protection from the disease. A survey was conducted among people age over 50 years $(n=1001)$ in Italy from October 2014 to April 2015 [38]. A total of 95\% of participants said they were aware of $\mathrm{HZ}$ disease. Most respondents (80\%) knew a person who had had $\mathrm{HZ}$ in the past, and $22 \%$ had already had HZ themselves. A total $91 \%$ did not know that there is a vaccine to prevent $\mathrm{HZ}$, and $85 \%$ said that they were generally in favor of $\mathrm{HZ}$ vaccination. This study proved the decisive role of primary care physicians in the decision-making process for $\mathrm{HZ}$ vaccination; $83 \%$ of survey respondents said they would receive vaccination if their primary care physician recommended it. The authors of a systematic review on the acceptance of $\mathrm{HZ}$ vaccination came to a similar conclusion [39]. According to the review, recommendation by a primary care physician is the decisive factor in high acceptance of a vaccine. Additional factors identified for successful vaccine implementation were cost reimbursement and awareness about HZ in the target population.
To estimate the implementation of vaccination recommendation in the target group of adults age 60 years and over in Germany, data were referenced on seasonal influenza vaccination coverage from invoicing data by the associations of statutory health insurance physicians [40] as well as data on the use of pneumococcal vaccines for older adults from the German health interview and examination survey for adults (DEGS) [41]. According to these data, nationwide influenza vaccination coverage has fallen from around $48 \%$ among people age $\geq 60$ years during the $2008 / 09$ and $2009 / 10$ seasons to less than $35 \%$ in the $2016 / 17$ season. Vaccination coverage appears to have stagnated since the 2012/2013 season at around one-third of all adults age 60 years and older who are vaccinated for seasonal influenza. The large spread among the federal states was also noteworthy (2016/17: $19.9 \%$ in Baden-Württemberg to $55.2 \%$ in Saxony-Anhalt). Based on the DEGS survey, $31.4 \%$ of people age $65-79$ years were vaccinated against pneumococcal disease in 2008-2011, women (33.2\%) somewhat more frequently than men $(29.3 \%)$. However, it remains unclear at this time whether these utilization data can be applied to $\mathrm{HZ}$ vaccination.

\section{Modelling the influence of the vaccination on $\mathrm{HZ}$ and $\mathrm{PHN}$ epidemiology in Germany}

\subsection{Methods}

A static Markov cohort model was used for analysis [42], updated specifically with regard to data on vaccine efficacy and vaccine-induced period of protection [43]. The model follows a simulated cohort of one million 50-year-olds to the end of their lives. It covers five conditions (health, death, HZ, PHN, and health after illness), and calculates a cycle length of 3 months based on duration of $\mathrm{HZ}$ illness and $\mathrm{PHN}$ definition. The age at vaccination was varied between 50 and 80 years in 5 year steps. In addition to the number of $\mathrm{HZ}(\mathrm{PHN})$ cases prevented by vaccination and the number needed to vaccinate (NNV) to prevent one $\mathrm{HZ}(\mathrm{PHN})$ case, health economics analyses were also conducted. Incremental cost-effectiveness ra- 


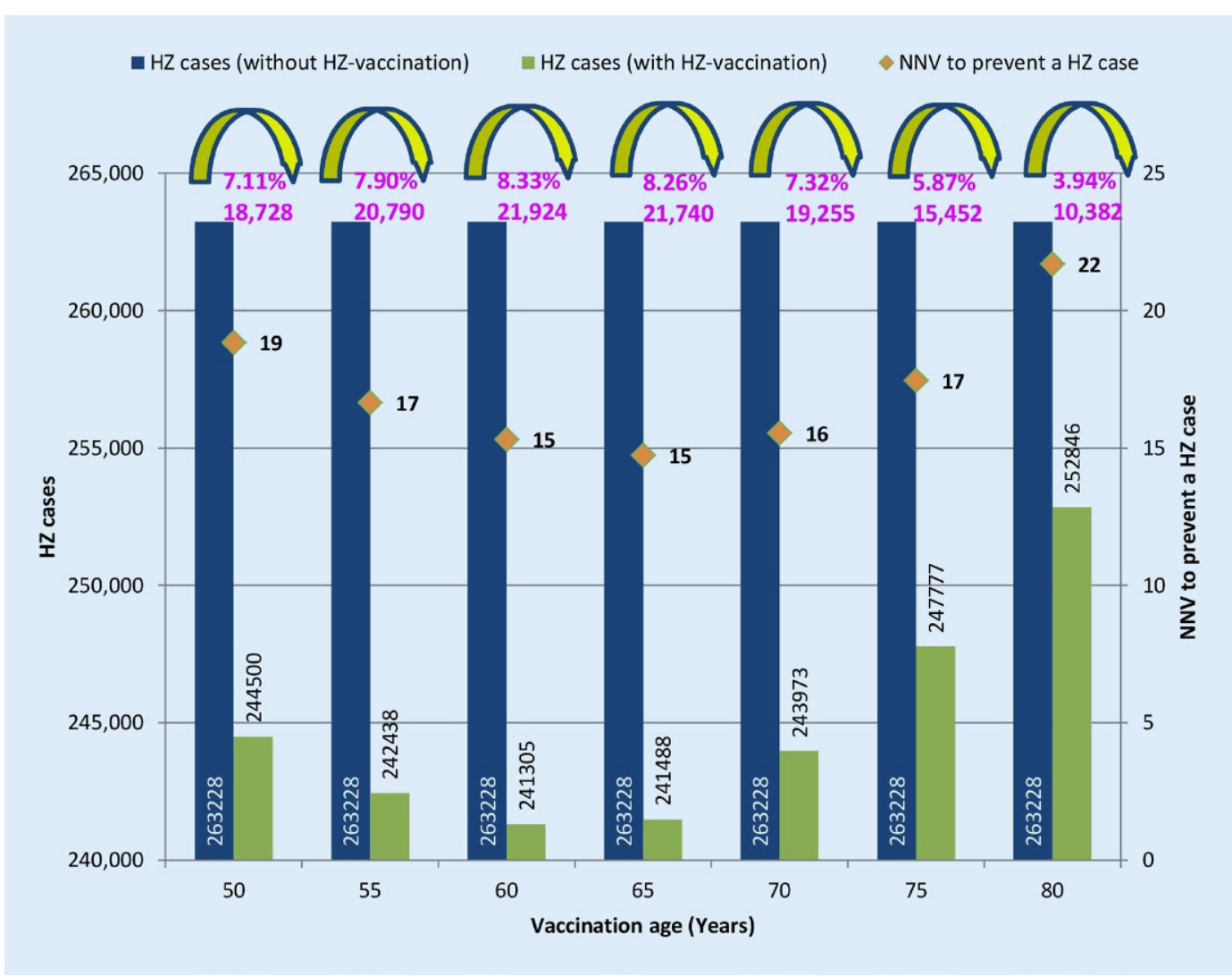

Fig. $10<$ Number of $\mathrm{HZ}$ cases with and without vaccination with the $\mathrm{HZ} /$ su inactivated vaccine and number needed to vaccinate (NNV) according to age at vaccination (vaccination coverage $35.5 \%$, cohort size 1,000,000, undiscounted) tios (ICER), with $€$ per $\mathrm{HZ}$ case prevented $(€ / \mathrm{HZ})$ and $€$ per QALY gained (€/QALY), were calculated. All analyses were calculated from a societal perspective, i.e., including costs for absenteeism from work. In addition to a base-case analysis (vaccination at age 60 years, assumed immunization costs of $€ 182$ per person vaccinated, $35.3 \%$ vaccination coverage, and $3 \%$ annual discount rate of costs and benefits), descriptive univariate and probabilistic sensitivity analyses were conducted, to identify the impact of uncertain input factors. The model was developed using the programming language $\mathrm{R}$ (The $\mathrm{R}$ Project for Statistical Computing, Vienna, Austria).

\subsection{Input data}

Data on epidemiology and on direct and indirect treatment costs of $\mathrm{HZ}$ and $\mathrm{PHN}$ were taken from invoicing data by the associations of statutory health insurance (SHI) physicians (KV) and from SHI funds in Germany $([5,44]$, Damm et al. (not yet published)). The costs for a complete vaccination series (two vaccine doses plus ad- ministration) were assumed to be $€ 182$ (https://www.rki.de/zoster-impfung). The theoretical price per dose (no information was available from the manufacturer at the time of modeling) was $€ 84$ and administration costs were $€ 7$. The data on vaccine effectiveness and vaccine-induced period of protection have been described above. Quality-of-life data for calculating QALYs were taken from patients with $\mathrm{HZ}$ or PHN disease in Canada [45].

\subsection{Results}

Of one million 50-year-olds without $\mathrm{HZ}$ vaccination in the model cohort, 260,000 will develop $\mathrm{HZ}$ in the remaining course of their lives (cf. 263,228/1,000,000=26.3\%), and 15,000 will develop PHN (cf. $15,325 / 1,000,000=1.5 \%)$. In the base-case scenario, 21,924 HZ cases $(\mathrm{NNV}=15)$, i.e., $8.33 \%$ of $\mathrm{HZ}$ cases that would occur without vaccination, could be prevented with the $\mathrm{HZ} / \mathrm{su}$ inactivated vaccine (- Fig. 10). A higher vaccination rate of e.g. $60 \%$ ( $80 \%$ ) could prevent 24,843 $(37,264) \mathrm{HZ}$ cases. The potential reduction in $\mathrm{HZ}$ cases varies according to age at vaccination. It is highest with vaccination at age 60 years $(8.3 \%)$ and lowest with vaccination at age 80 years (3.9\%) (• Fig. 10). The lowest NNV was achieved with vaccination at the ages of 60 and 65 years, with 15 for each. According to the model, the most PHN cases can be prevented with vaccination at the age of 70 years $(9.9 \%)$, followed by $9.8 \%$ for vaccination at age 65 years and $9 \%$ for vaccination at age 60 years (- Fig. 11). The NNV to prevent one PHN case also varies according to age at vaccination, and ranges from 421 for vaccination at age 50 years to 197 for vaccination at age 70 years (• Fig. 11).

Vaccination with the HZ/su inactivated vaccine leads to ICERs of $€ 1774 / \mathrm{HZ}$ and $€ 23,934 / \mathrm{QALY}$ in the base-case scenario. Vaccination at age 65 years appears to be the most cost-effective, but the difference between vaccination at age 60 years is small (• Fig. 12).

In other sensitivity analyses, it was shown that especially the vaccine-induced period of protection, price of the vaccine, and recurrence rate of $\mathrm{HZ}$ have the greatest impact on base-case results. If theoretical lifelong vaccine protection is 


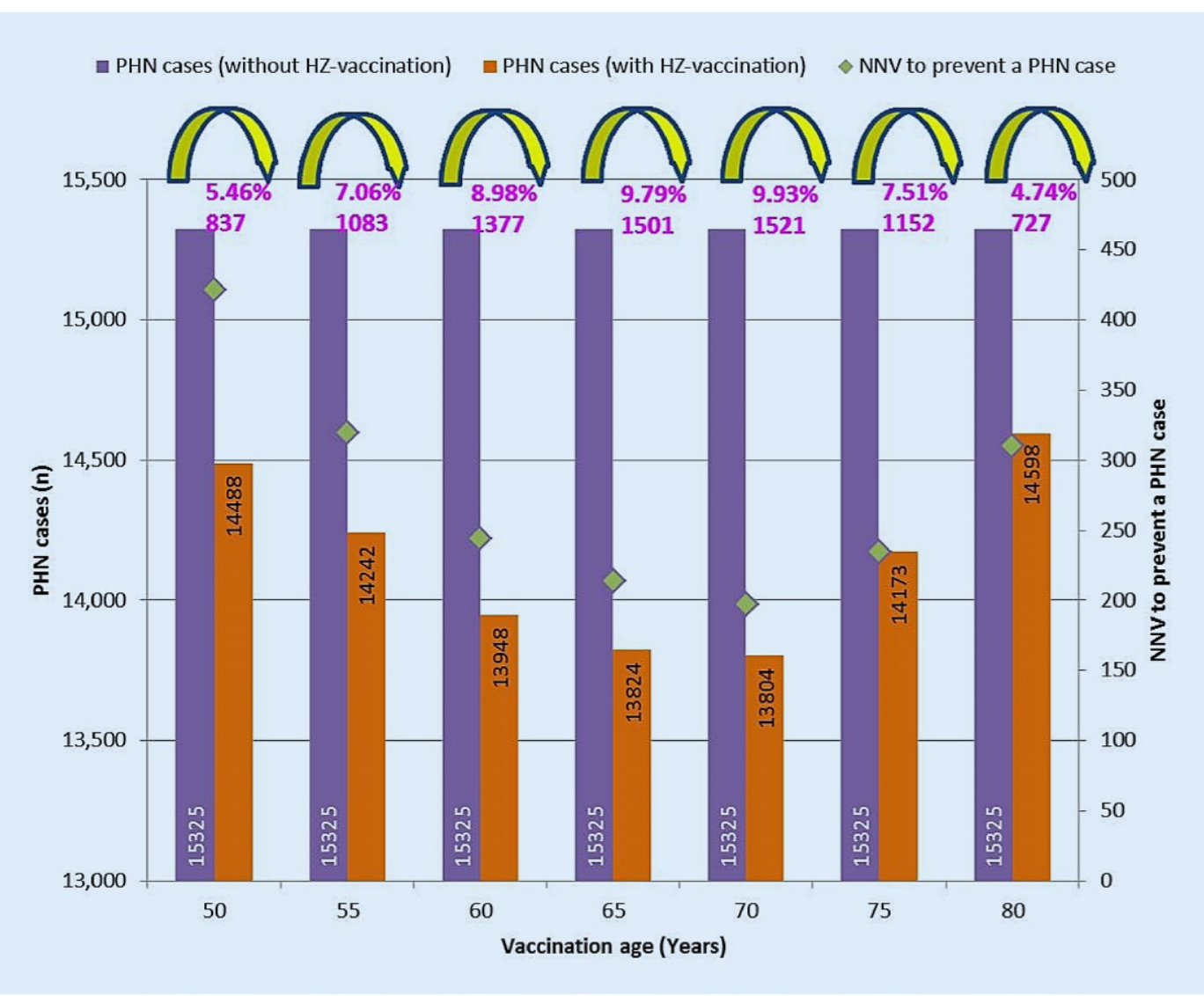

Fig. $11 \triangleleft$ Number of PHN cases with and without vaccination with the $\mathrm{HZ} /$ su inactivated vaccine and NNV according to age at vaccination (vaccination coverage $35.5 \%$, cohort size $1,000,000$, undiscounted)

assumed, the ICER falls to $€ 8523$ /QALY for vaccination at age 60 years. Assuming a period of protection of only 5 years leads to an ICER of $€ 86,678 /$ QALY, if the immunization costs fall from $€ 182$ to $€ 100$, the resulting ICER is $€ 11,437 /$ QALY; if these costs rise to $€ 282$, the ICER is then $€ 39,173 /$ QALY (• Fig. 12).

\subsection{Conclusions from modelling}

In a cohort of one million 50-year-olds that were followed up until the end of their life, modeling the epidemiological effects of $\mathrm{HZ}$ vaccination revealed that vaccination at age 60 would prevent most $\mathrm{HZ}$ cases and vaccination at age 70 would prevent most PHN cases. The health economic model showed the best cost-effectiveness with vaccination at age 65 years, which was only slightly better than vaccination at 60 years of age. NNV was lowest for vaccination at ages 60 and 65 years, with the same values. As results on VE against PHN are based on very few cases (see $\bullet$ Table 2) and prevention of $\mathrm{HZ}$ is the precondition for preventing $\mathrm{PHN}$, and because vaccination at age 60 years would prevent most $\mathrm{HZ}$ cases according to the model, 60 years of age seem to be the best age for vaccination, from an epidemiologic point of view.

\section{Evaluation of the vaccination recommendation}

\subsection{Epidemiology monitoring}

From invoicing data of the associations of SHI physicians, diagnostic data are available for monitoring the epidemiology of $\mathrm{HZ}$ and $\mathrm{PHN}$ in individuals insured under SHI [5]. Information on $\mathrm{HZ}$ and PHN epidemiology before the introduction of vaccination is available from the same data source, such that a potential effect of vaccination at population level can be assessed very well using the same data source. Furthermore, $\mathrm{HZ}$ is a notifiable disease in the federal states of Brandenburg and Saxony, according to state regulations; thus, further population-based epidemiological data are available for the comparison of age specific HZ-incidenc- es in periods pre- and post-introduction of vaccination.

\subsection{Monitoring of adverse drug reactions}

Surveillance systems have been established in Germany at the Paul Ehrlich Institute for Vaccines and Biomedicines (PEI) for the spontaneous recording of suspected cases of possible adverse events after vaccination. According to the German Medicines Act (Arzneimittelgesetz, AMG), possible side effects of vaccines must be reported by the marketing authorization holder and/or pharmaceutical company. Doctors are also required to report suspected cases of vaccine complications in accordance with the Protection Against Infection Act (IfSG) [46, 47]. Examples of how these data can be used include conducting observed versus expected analyses and examination of whether certain events occur more frequently among those recently vaccinated than would be expected, compared with background incidence for the age group; 


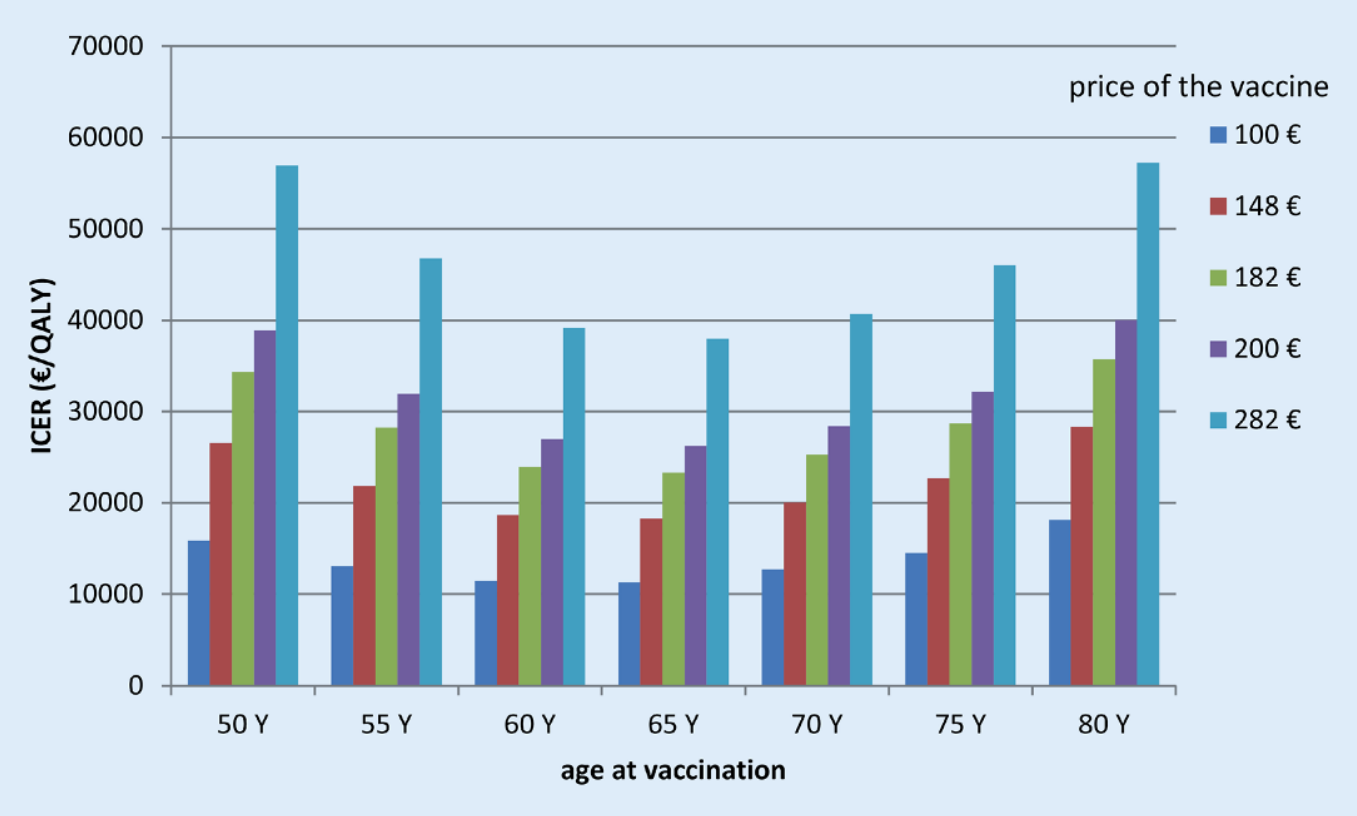

Fig. $12<$ Costs per quality-adjusted life year (QALY) gained according to vaccine price and age at vaccination in addition, the data can be used in quantitative methods, such as the proportional reporting ratio (PRR).

If illnesses occur in temporal correlation to vaccinations, it is important to distinguish between a causal link and coincidental events. For that reason, researchers at the Robert Koch Institute (RKI) and PEI are conducting a joint project to determine estimators of the background incidence in selected immune-mediated diseases, orthostatic hypotension, myocardial infarction, stroke, and sudden death in adults age $\geq 50$ years in Germany. Based on these estimators, the expected number of newly occurring diseases in a certain time interval, independent of vaccination with the HZ/su inactivated vaccine, can be calculated. This can be used in a comparison with the observed number of these diseases after introduction of the HZ/su inactivated vaccine, to generate warnings should the comparison point to an elevated number of rare adverse side effects.

\subsection{Vaccination coverage monitoring}

The aforementioned invoicing data from the associations of SHI physicians on vaccinations and relevant diagnoses (HZ, PHN) can be used and analyzed [48]. This facilitates the determination of nationwide vaccination coverage among people insured under SHI (around 85\% of the population). These data are available with a 6-month delay. In addition, incidences of the target diseases can be determined according to age and risk group by the same data source, such that the implementation of $\mathrm{HZ}$ vaccination recommendations and their impact can be evaluated using this system.

\subsection{Summary evaluation of the vaccination recommendation}

For the ongoing evaluation of $\mathrm{HZ}$ vaccination recommendations, various surveillance and monitoring systems have been implemented that permit the continuous recording of data on $\mathrm{HZ}$ and $\mathrm{PHN}$ epidemiology, the occurrence of adverse effects of vaccination, and $\mathrm{HZ}$ vaccination coverage. Data collection via these systems was in place prior to the introduction of $\mathrm{HZ}$ vaccination. Thus, reliable evaluation of the vaccination recommendation is possible by comparing data between the pre- and post-vaccination phases. Using the available data, it is possible to assess vaccine effectiveness after vaccination by age and risk groups as well as over time. In addition, international publications are continuously reviewed, which report on vaccine effectiveness, duration of vaccine-induced protection, and vaccine safe- ty; the results will be analyzed and compared with the data from Germany. This will contribute to answering any remaining questions, such as how long the vaccine will protect against $\mathrm{HZ}$ as a clinical endpoint.

\section{Corresponding address}

\section{A. Siedler}

Immunization Unit, Robert Koch Institute

Berlin, Germany

SiedlerA@rki.de

Acknowledgements. We thank Analisa Avila, ELS, of Edanz Group (www.edanzediting.com/ac) for editing a draft of this manuscript.

Conflict of interest. E. Garbe participated in two studies on the epidemiology of herpes zoster and PHN that were financially supported one by SPMSD and the other by GSK. A. Siedler, J. Koch, H. Hengel, R. von Kries, T. Ledig, T. Mertens, F. Zepp and $\mathrm{K}$. Überla declare that they have no competing interests. 


\section{References}

1. Siedler A, Koch J, Ultsch B, Garbe E, von Kries R, Ledig T, Mertens T, Überla K, Zepp F, Hengel H (2017) Background paper to the decision not to recommend a standard vaccination with the live attenuated herpes zoster vaccine for the elderly in Germany. Bundesgesundheitsbl 60(60):1162-1179

2. German Standing Committee on Vaccination (STIKO) (2011) Standardvorgehensweise (SOP) der Ständigen Impfkommission (STIKO) für die systematische Entwicklung von Impfempfehlungen. https://www.rki.de/EN/Content/infections/ Vaccination/methodology/SOP.pdf?_blob=publicationFile. Accessed 28 Mar 2012 (Standard Operating Procedure of the German Standing Committee on Vaccinations (STIKO) for the systematic development of vaccination recommendations)

3. Ständige Impfkommission (STIKO) (2017) Ständige Impfkommission (STIKO). Wissenschaftliche Begründung für die Entscheidung, die Herpes zoster Lebendimpfung nicht als Standardimpfung zu empfehlen. Epid Bull 36:391-410

4. Hillebrand $K$, Bricout $H$, Schulze-Rath $R$, Schink T, Garbe $E$ (2015) Incidence of herpes zoster and its complications in Germany, 2005-2009. J Infect 70(2):178-186

5. Ultsch B, Siedler A, Rieck T, Reinhold T, Krause G, Wichmann O (2011) Herpes zoster in Germany: quantifying the burden of disease. Bmc Infect Dis 11:173

6. Forbes HJ, Bhaskaran K, Thomas SL, Smeeth L, Clayton T, Langan SM (2014) Quantification of risk factors for herpes zoster: population based case-control study. BMJ 348. https:// doi.org/10.1136/bmj.g2911

7. European Medicines Agency (EMA) (2018) Summary of product characteristics of Shingrix (herpes zoster vaccine, recombinant, adjuvanted). https://www.ema.europa.eu/documents/product-information/shingrix-epar-product-information_en.pdf. Accessed 10 June 2018

8. Didierlaurent AM, Laupèze $B$, Di Pasquale $A$, Hergli N, Collignon C, Garçon N (2017) Adjuvant system AS01: helping to overcome the challenges of modern vaccines. Expert Rev Vaccines 16(1):55-63

9. Glaxo Smith Kline (GSK) (2018) Package leaflet Shingrix(R), 2018 [updated March 2018; cited 2018 16. https://www.gsk-arzneimittel.de/assetManager.xm?action=getFi\&id=13715870. Accessed 17 Oct 2018

10. Moher D, Liberati A, Tetzlaff J, Altman DG, Group P (2009) Preferred reporting items for systematic reviews and meta-analyses: the PRISMA statement. BMJ 339:b2535

11. Higgins JP, Altman DG, Gotzsche PC, Juni P, Moher D, Oxman AD et al (2011) The Cochrane Collaboration's tool for assessing risk of bias in randomised trials. BMJ 343:d5928

12. Guyatt $G H, O x m a n A D$, Schunemann HJ, Tugwell P, Knottnerus A (2011) GRADE guidelines: a new series of articles in the Journal of Clinical Epidemiology. J Clin Epidemiol 64(4):380-382

13. Guyatt $G H$, Oxman $A D$, Vist $G E$, Kunz R, Falck-Ytter Y, Alonso-Coello P et al (2008) GRADE: an emerging consensus on rating quality of evidence and strength of recommendations. BMJ 336(7650):924-926

14. Cunningham AL, Lal H, Kovac M, Chlibek R, Hwang SJ, Diez-Domingo J et al (2016) Efficacy of the
Herpes Zoster Subunit Vaccine in Adults 70 Years of Age or Older. N Engl J Med 375(11):1019-1032

15. Lal H, Cunningham AL, Godeaux O, Chlibek R, Diez-Domingo J, Hwang SJ et al (2015) Efficacy of an adjuvanted herpes zoster subunit vaccine in older adults. N Engl J Med 372(22):2087-2096

16. Chlibek R, Smetana J, Pauksens K, Rombo L, Van den Hoek JA, Richardus JH et al (2014) Safety and immunogenicity of three different formulations of an adjuvanted varicella-zoster virus subunit candidate vaccine in older adults: a phase II, randomized, controlled study. Vaccine 32(15):17451753

17. Chlibek R, Pauksens K, Rombo L, van Rijckevorsel G, Richardus JH, Plassmann G et al (2016) Long-term immunogenicity and safety of an investigational herpes zoster subunit vaccine in older adults. Vaccine 34(6):863-868

18. Pauksens K, Volpe S, Schwarz TF, Smetana J, Toursarkissian N, Rombo L et al (2017) Persistence of Immune Response to an Adjuvanted VaricellaZoster Virus Subunit Candidate Vaccine for up to Year 9 in Older Adults. Open Forum Infect Dis 4(Suppl 1):415

19. Chlibek R, Bayas JM, Collins H, de la Pinta ML, Ledent E, Mols JF et al (2013) Safety and immunogenicity of an AS01-adjuvanted varicella-zoster virus subunit candidate vaccine against herpes zoster in adults $>=50$ years of age. J Infect Dis 208(12):1953-1961

20. EMA Shingrix- EPAR -Assesment report 2018. https://www.ema.europa.eu/documents/product-information/shingrix-epar-product-information_en.pdf. Accessed 17 Oct 2018

21. Lal H, Poder A, Campora L, Geeraerts B, Oostvogels L, Vanden Abeele C et al (2018) Immunogenicity, reactogenicity and safety of 2 doses of an adjuvanted herpes zoster subunit vaccine administered 2, 6 or 12 months apart in older adults: Results of a phase III, randomized, open-label, multicenter study. Vaccine 36(1):148-154

22. Schwarz TF, Aggarwal N, Moeckesch B, Schenkenberger I, Claeys C, Douha M et al (2017) Immunogenicity and safety of an adjuvanted herpes zoster subunit vaccine co-administered with seasonal influenza vaccine in adults aged 50 years and older. J Infect Dis. https://doi.org/10.1093/infdis/ jix481

23. Marechal C, Lal H, Poder A, Ferguson M, Enweonye I, Heineman TC et al (2018) Immunogenicity and safety of the adjuvanted recombinant zoster vaccine co-administered with the 23 -valent pneumococcal polysaccharide vaccine in adults $>/=50$ years of age: $\mathrm{A}$ randomized trial. Vaccine 36(29):4278-4286

24. Godeaux O, Kovac M, Shu D, Grupping K, Campora L, Douha M et al (2017) Immunogenicity and safety of an adjuvanted herpes zoster subunit candidate vaccine in adults $>/=50$ years of age with a prior history of herpes zoster: A phase III, non-randomized, open-label clinical trial. Hum Vaccin Immunother 13(5):1051-1058

25. Grupping K, Campora L, Douha M, Heineman TC, Klein NP, Lal H et al (2017) Immunogenicity and Safety of the HZ/su Adjuvanted Herpes Zoster Subunit Vaccine in Adults Previously Vaccinated with a Live-Attenuated Herpes Zoster Vaccine. J Infect Dis 216(11):1343-1351. https:// doi.org/10.1093/infdis/jix482

26. Vink P, Shiramoto M, Ogawa M, Eda M, Douha M, Heineman T et al (2017) Safety and immunogenicity of a Herpes Zoster subunit vaccine in Japanese population aged $>/=50$ years when administered subcutaneously vs. intramuscularly. Hum Vaccin Immunother 13(3):574-578

27. Rogers JE, Cumpston A, Newton M, Craig M (2011) Onset and complications of varicella zoster reactivation in the autologous hematopoietic cell transplant population. Transpl Infect Dis 13(5):480-484

28. Schuchter LM, Wingard JR, Piantadosi S, Burns WH, Santos GW, Saral R (1989) Herpes zoster infection after autologous bone marrow transplantation. Blood 74(4):1424-1427

29. Stadtmauer EA, Sullivan KM, Marty FM, Dadwal SS, Papanicolaou GA, Shea TC et al (2014) A phase $1 / 2$ study of an adjuvanted varicella-zoster virus subunit vaccine in autologous hematopoietic cell transplant recipients. Blood 124(19):2921-2929

30. De la Serna J Efficacy and Safety of an Adjuvanted Herpes Zoster Subunit Vaccine in Autologous Hematopoietic Stem Cell Transplant Recipients 18 Years of Age or Older: First Results of the Phase 3 Randomized, Placebo-Controlled ZOE-HSCT Clinical Trial. https://bmt.confex.com/tandem/2018/ meetingapp.cgi/Paper/11724 (BMT Tandem Meeting, Salt Lake City, Utah ,21-25 February, 2018)

31. Donahue JG, Choo PW, Manson JE, Platt R (1995) The incidence of herpes zoster. Arch Intern Med 155(15):1605-1609

32. Buchbinder SP, Katz MH, Hessol NA, Liu JY, O'Malley PM, Underwood R et al (1992) Herpes zoster and human immunodeficiency virus infection. J Infect Dis 166(5):1153-1156

33. Moanna A, Rimland D (2013) Decreasing Incidence of Herpes Zoster in the Highly Active Antiretroviral Therapy Era. Clin Infect Dis 57(1):122-125

34. Berkowitz EM, Moyle G, Stellbrink HJ, Schurmann D, Kegg S, Stoll M et al (2015) Safety and immunogenicity of an adjuvanted herpes zoster subunit candidate vaccine in HIV-infected adults: a phase $1 / 2$ a randomized, placebo-controlled study. $J$ Infect Dis 211(8):1279-1287

35. Vink P (2017) the Zoster-028 Study G. Immunogenicity and Safety of a Candidate Subunit Adjuvanted Herpes Zoster Vaccine in Adults with Solid Tumors Vaccinated Before or During Immunosuppressive Chemotherapy Treatment: A Phase II/III, Randomized Clinical Trial. Open Forum Infect Dis 4(Suppl 1):417-S8

36. L Oostvogels. Immunogenicity and Safety of an Adjuvanted Herpes Zoster Subunit Candidate Vaccine in Adults with Hematologic Malignancies: A Phase III, Randomized Clinical Trial,. IDWeek, San Diego, CA, USA, . 4-8 October, 2017;Abstract.

37. Vink P (2017) Immunogenicity and Safety of a Candidate Subunit Adjuvanted Herpes Zoster Vaccine (HZ/su) in Adults Post Renal Transplant: a Phase III Randomized Clinical Trial. Open Forum Infect Dis 4(Suppl 1):417

38. Valente N, Lupi S, Stefanati A, Cova M, Sulcaj N, Piccinni L et al (2016) Evaluation of the acceptability of a vaccine against herpes zoster in the over 50 years old: an Italian observational study. BMJ Open 6(10):e11539

39. Damm O, Witte J, Greiner W (2015) A Systematic Review of Herpes Zoster Vaccine Acceptance. Value Health 18(7):A592

40. Rieck T, Feig M, Siedler A, Wichmann O (2018) Aktuelles aus der KV-Impfsurveillance - Impfquoten ausgewählter Schutzimpfungen in Deutschland. Epid Bull 01(2018):1-14. https://doi.org/10.17886/ EpiBull-2018-001.3

41. Poethko-Müller C, Schmitz R (2013) Impfstatus von Erwachsenen in Deutschland. Bundesgesundheitsbl 56:845-857. https://doi.org/10.1007/s00103013-1693-6 
42. Ultsch B, Weidemann F, Reinhold T, Siedler A, Krause G, Wichmann O (2013) Health economic evaluation of vaccination strategies for the prevention of herpes zoster and postherpetic neuralgia in Germany. Bmc Health Serv Res 13:359

43. Ultsch B, Weidemann F, Koch J, Siedler A (2017) Projektbericht: Modellierung von epidemiologischen und gesundheitsökonomischen Effekten von Impfungen zur Prävention von Herpes zoster. Robert Koch Institute, Berlin. https://www.rki.de/ DE/Content/Infekt/Impfen/ImpfungenAZ/Zoster/ Modellierung_Zoster_Impfung.pdf?_blob=publicationFile (Project report: Modeling of epidemiological and health economic effects of vaccinations for the prevention of herpes zoster)

44. Ultsch B, Koster I, Reinhold T, Siedler A, Krause G, Icks A et al (2013) Epidemiology and cost of herpes zoster and postherpetic neuralgia in Germany. Eur J Health Econ 14(6):1015-1026

45. Drolet M, Brisson M, Schmader KE, Levin MJ, Johnson R, Oxman MN et al (2010) The impact of herpes zoster and postherpetic neuralgia on health-related quality of life: a prospective study. CMAJ 182(16):1731-1736

46. Weisser K, Meyer C, Petzold D, Mentzer D, Keller-Stanislawski B (2007) Adverse drug reactions following immunization in Germany pursuant to the German Infection Protection Act and the German Medicinal Products Act from January 1, 2004 to December 31, 2005. Bundesgesundheitsblatt Gesundheitsforschung Gesundheitsschutz 50(11):1404-1417

47. Keller-Stanislawski B, Heuss N, Meyer C (2004) Adverse events following immunisation in Germany from 1.1.2001 to 31.12.2003. Bundesgesundheitsblatt Gesundheitsforschung Gesundheitsschutz 47(12):1151-1164

48. Siedler A, Rieck T, Reuss A, Walter D, Poggensee G, Poethko-Muller C et al (2012) Estimating vaccination coverage in the absence of immunisation registers-the German experience. Euro Surveill 17(17). https://doi.org/10.2807/ese.17.17.20152en 